\title{
OPTIMAL CONTROL OF THE TWO-DIMENSIONAL EVOLUTIONARY NAVIER-STOKES EQUATIONS WITH MEASURE VALUED CONTROLS*
}

\author{
EDUARDO $\mathrm{CASAS}^{\dagger}$ AND KARL KUNISCH ${ }^{\ddagger}$
}

\begin{abstract}
In this paper, we consider an optimal control problem for the two-dimensional evolutionary Navier-Stokes system. Looking for sparsity, we take controls as functions of time taking values in a space of Borel measures. The cost functional does not involve directly the control but we assume some constraints on them. We prove the well-posedness of the control problem and derive necessary and sufficient conditions for local optimality of the controls.
\end{abstract}

Key words. Navier-Stokes equations, Borel measures, sparsity, first and second order optimality conditions

AMS subject classifications. 35Q30, 49J20, 49J52, 49K20, 49K40

DOI. $10.1137 / 20 \mathrm{M} 1351400$

1. Introduction. In this paper we investigate the following optimal control problem

$$
\text { (P) } \min _{\mathbf{u} \in \mathbf{U}_{\text {ad }}} J(\mathbf{u})=\frac{1}{2} \int_{Q}\left|\mathbf{y}_{\mathbf{u}}(x, t)-\mathbf{y}_{d}(x, t)\right|^{2} d x d t,
$$

where $\mathbf{U}_{\mathrm{ad}}=\left\{\mathbf{u} \in L^{\infty}(0, T ; \mathbf{M}(\omega)):\|\mathbf{u}(t)\|_{\mathbf{M}(\omega)} \leq \gamma\right.$ for a.a. $\left.t \in(0, T)\right\}$ with $0<$ $\gamma<\infty$, and $\mathbf{y}$ and $\mathbf{u}$ are related by the Navier-Stokes system

$$
\left\{\begin{array}{l}
\frac{\partial \mathbf{y}}{\partial t}-\nu \Delta \mathbf{y}+(\mathbf{y} \cdot \nabla) \mathbf{y}+\nabla \mathfrak{p}=\mathbf{f}_{0}+\chi_{\omega} \mathbf{u} \text { in } Q=\Omega \times I, \\
\operatorname{div} \mathbf{y}=0 \text { in } Q, \mathbf{y}=0 \text { on } \Sigma=\Gamma \times I, \mathbf{y}(0)=\mathbf{y}_{0} \text { in } \Omega
\end{array}\right.
$$

Here, $I=(0, T)$ with $0<T<\infty, \Omega$ denotes a bounded domain in $\mathbb{R}^{2}$ with a $C^{3}$ boundary $\Gamma$, and $\omega$ is a relatively closed subset of $\Omega$. We denote $\mathbf{M}(\omega)=M(\omega) \times M(\omega)$, where $M(\omega)$ is the space of real and regular Borel measures in $\omega$. In the cost functional $J$, the target $\mathbf{y}_{d} \in \mathbf{L}^{2}(Q)$ is fixed. Regarding the state equation, $\nu>0$ is the kinematic viscosity coefficient, $\chi_{\omega} \mathbf{u}$ denotes the extension of $\mathbf{u}$ by zero outside $\omega$, and $\mathbf{f}_{0}$ is a given element of $L^{q}\left(I, \mathbf{W}^{-1, p}(\Omega)\right)$ with $\mathbf{W}^{-1, p}(\Omega)=W^{-1, p}(\Omega) \times W^{-1, p}(\Omega)$, where

$$
\frac{4}{3} \leq p<2 \quad \text { and } \quad q>\frac{2 p}{p-1}
$$

are fixed. Observe that the previous assumptions imply that $q>4$. For the initial condition we can take $\mathbf{y}_{0} \in \mathbf{W}_{0}^{1, p}(\Omega)$ such that $\operatorname{div} \mathbf{y}_{0}=0$. A more general choice for $\mathbf{y}_{0}$ will be given later.

\footnotetext{
*Received by the editors July 8, 2020; accepted for publication (in revised form) March 12, 2021; published electronically June 17, 2021.

https://doi.org/10.1137/20M1351400

Funding: The first author was supported by Spanish Ministerio de Economía, Industria y Competitividad under research project MTM2017-83185-P. The second author was supported by the ERC advanced grant 668998 (OCLOC) under the EU's H2020 research program.

${ }^{\dagger}$ Departamento de Matemática Aplicada y Ciencias de la Computación, E.T.S.I. Industriales y de Telecomunicación, Universidad de Cantabria, 39005 Santander, Spain (eduardo.casas@unican.es).

${ }^{\ddagger}$ Institute for Mathematics and Scientific Computing, University of Graz, Heinrichstrasse 36, A8010 Graz, Austria (karl.kunisch@uni-graz.at).
} 
Our motivation for the analysis of measure-valued controls is two-fold. On the one hand there it is the genuine interest in low-order regularity of the controls, on the other hand it relates to their sparsity promoting structure. Indeed, it has been observed and analyzed in much previous work that the optimal controls are typically zero over subsets of the domain, whereas they would simply be "small," but not zero, if they would be replaced by a control in a Hilbert space, for example. We refer, exemplarily to the work in $[6,8,23]$, which treats these phenomena for equations of diffusion type as well as for wave equations. In these papers the sparsity promoting terms is part of the cost, whereas in [14] the measure valued term appears as a constraint like in $\mathbf{U}_{\mathrm{ad}}$ above. It should also be mentioned that in case the measure-valued setting is replaced by an $L^{1}$ formulation together with $L^{2}$ constraints or penalties, again sparsity phenomena occur, but the optimal controls are, of course, functions in this case rather than measures $[11,21]$.

In the literature, the optimal control of the Navier-Stokes equations has received much attention; we refer exemplarily to $[1,4,16,17,22,30]$, and the monograph [20] and the survey [7]. The controls are always considered as functions in these contributions. Apparently the only work on measure valued optimal controls in the case of the Navier-Stokes equations is [13] which treats the stationary case.

For evolutionary Navier-Stokes equations with forcing functions of low regularity, allowing for measure-valued forcing, very little analysis has been carried out even for the state equation by itself. We are only aware of [26], where the right-hand side in (1.1) is chosen in $W^{1, \infty}\left(I ; \mathbf{W}^{-1, p}(\Omega)\right)$, with $\mathbf{W}^{-1, p}(\Omega)=\bigotimes_{i=1}^{d} W^{-1, p}(\Omega), d \in\{2,3\}$, and $p \in\left(\frac{d}{2}, 2\right]$. It is mentioned there that likely the result is not optimal. In our previous work [15] we have obtained the necessary well-posedness results for (1.1) which are required for the study of optimal control problems. Thus the current work is the first one which considers optimal control for evolutionary Navier-Stokes equations with measure-valued controls.

When formulating optimal control problems some restrictions on the class of admissible controls are essential to guarantee existence of minimizers to be obtained by the standard method of the calculus of variations. Such restrictions are also well motivated by applications. One possible choice consists in adding a properly chosen control cost to the cost-functional $J$ in $(\mathrm{P})$. In our case it could be a term of the form $\frac{\beta}{q} \int_{0}^{T}\|\mathbf{u}(t)\|_{\mathbf{M}(\omega)}^{q} d t$, where $\beta$ is a positive weight. For technical reasons $q=2$ seems not to be possible, since it does not imply sufficient temporal regularity on the class of admissible controls. From the analytical point of view it would suffice to take $q>4$. But we prefer to rather work with pointwise constraints in time. In this way we arrive at the class $\mathbf{U}_{\mathrm{ad}}$ and the problem formulation chosen in $(\mathrm{P})$. This choice of temporal pointwise constraints also poses new challenges in deriving both necessary and sufficient second order optimality conditions, regardless of the measure-valued norm in space.

Let us comment further on the norm in $\mathbf{M}(\omega)$ appearing in $(\mathrm{P})$. First, we recall that $M(\omega)$ is a Banach space when endowed with the norm

$$
\|u\|_{M(\omega)}=\sup _{\|\phi\|_{C_{0}(\omega)} \leq 1} \int_{\omega} \phi(x) d u(x)=|u|(\omega)
$$

where $C_{0}(\omega)=\{\phi \in C(\bar{\omega}): \phi(x)=0 \forall x \in \partial \omega \cap \Gamma\}$ is a separable Banach space, and $|u|$ represents the total variation measure of $u$; see [25, page 130]. Note that $C_{0}(\omega) \neq C(\bar{\omega})$ only in the case that $\bar{\omega}$ has a nonempty intersection with $\Gamma$. 
For vector-valued measures we define

$$
\|\mathbf{u}\|_{\mathbf{M}(\omega)}=\max \left(\left\|u_{1}\right\|_{M(\omega)},\left\|u_{2}\right\|_{M(\omega)}\right),
$$

which makes $\mathbf{M}(\omega)$ a Banach space. It is the dual space of $\mathbf{C}_{0}(\omega)=C_{0}(\omega) \times C_{0}(\omega)$ when it is endowed with the norm $\|\phi\|_{\mathbf{C}_{0}(\omega)}=\left\|\phi_{1}\right\|_{C_{0}(\omega)}+\left\|\phi_{2}\right\|_{C_{0}(\omega)}$.

Hereafter we denote by $L^{\infty}(I ; \mathbf{M}(\omega))$ the space of weakly measurable functions $\mathbf{u}:(0, T) \longrightarrow \mathbf{M}(\omega)$ satisfying $\|\mathbf{u}\|_{L^{\infty}(I ; \mathbf{M}(\omega))}=\operatorname{ess}_{\sup _{t \in I}}\|\mathbf{u}(t)\|_{\mathbf{M}(\omega)}<\infty$. This norm makes $L^{\infty}(I ; \mathbf{M}(\omega))$ a Banach space and guarantees that it can be identified with the dual of $L^{1}\left(I ; \mathbf{C}_{0}(\omega)\right)$, where the duality relation is given by

$$
\langle\mathbf{u}, \mathbf{z}\rangle_{L^{\infty}(I ; \mathbf{M}(\omega)), L^{1}\left(I ; \mathbf{C}_{0}(\omega)\right)}=\int_{0}^{T}\langle\mathbf{u}(t), \mathbf{z}(t)\rangle_{\mathbf{M}(\omega), \mathbf{C}_{0}(\omega)} d t .
$$

The reader is referred to [18, section 8.14.1 and Proposition 8.15.3] for the different notions of measurability and [18, Theorem 8.18.2] for the duality identification. (The distinction between weak and strong measurability is not required for the space $L^{1}\left(I ; \mathbf{C}_{0}(\omega)\right)$ because $\mathbf{C}_{0}(\omega)$ is separable and hence both notions are equivalent; see [18, Theorem 8.15.2].) Observe that $L^{\infty}(I ; \mathbf{M}(\omega))$ is a subspace of $L^{\infty}\left(I ; \mathbf{W}^{-1, p}(\Omega)\right)$ for every $p<2$. Indeed, the embedding $\mathbf{W}_{0}^{1, p^{\prime}}(\Omega) \subset \mathbf{C}_{0}(\Omega) \subset \mathbf{C}_{0}(\omega)$ implies that the duality $\langle\mathbf{u}(t), \mathbf{z}\rangle$ is well defined for every $\mathbf{u} \in L^{\infty}(I ; \mathbf{M}(\omega))$ and $\mathbf{z} \in \mathbf{W}_{0}^{1, p^{\prime}}(\Omega)$, and we have

$$
\begin{aligned}
\left|\langle\mathbf{u}(t), \mathbf{z}\rangle_{\mathbf{M}(\omega), \mathbf{C}_{0}(\omega)}\right| & \leq\|\mathbf{u}(t)\|_{\mathbf{M}(\omega)}\|\mathbf{z}\|_{\mathbf{C}_{0}(\omega)} \\
& \leq C_{p, \Omega}\|\mathbf{u}(t)\|_{\mathbf{M}(\omega)}\|\mathbf{z}\|_{\mathbf{W}_{0}^{1, p^{\prime}}(\Omega)} \leq C_{p, \Omega}\|\mathbf{u}\|_{L^{\infty}(I ; \mathbf{M}(\omega))}\|\mathbf{z}\|_{\mathbf{W}_{0}^{1, p^{\prime}}(\Omega)}
\end{aligned}
$$

for a.a. $t \in I$ and a constant $C_{p, \Omega}$ depending only on $p$ and $\Omega$. Analogously, we have that $L^{q}(I ; \mathbf{M}(\omega))$ is a Banach space for the norm

$$
\|\left.\mathbf{u}\right|_{L^{q}(I ; \mathbf{M}(\omega))}=\left(\int_{0}^{T}\|\mathbf{u}\|_{\mathbf{M}(\omega)}^{q} d t\right)^{1 / q}
$$

dual of $L^{q^{\prime}}\left(I ; \mathbf{C}_{0}(\omega)\right)$. Obviously, the embedding $L^{\infty}(I ; \mathbf{M}(\omega)) \subset L^{q}(I ; \mathbf{M}(\omega))$ holds. The right-hand side of the state equation, $\mathbf{f}_{0}+\chi_{\omega} \mathbf{u}$, is well defined as an element of $L^{q}\left(I ; \mathbf{W}^{-1, p}(\Omega)\right)$ for every $\mathbf{u} \in L^{q}(I ; \mathbf{M}(\omega))$.

Structure of paper. In the following section, well-posed results on the state equation relevant for the remainder of the paper are summarized. Here we can rely on results from [15]. Existence of solutions to (P) and first-order optimality conditions are the contents of section 3. Necessary and sufficient second-order optimality conditions will be given in section 4 . This requires further detailed analysis of the state equations and its linearization in function spaces of low regularity.

\section{Notation.}

In this paper, we denote $\mathbf{W}_{0}^{1, s}(\Omega)=W_{0}^{1, s}(\Omega) \times W_{0}^{1, s}(\Omega)$ for $s \in(1, \infty)$, and we choose as the norm in $\mathbf{W}_{0}^{1, s}(\Omega)$

$$
\|\mathbf{y}\|_{\mathbf{W}_{0}^{1, s}(\Omega)}=\|\nabla \mathbf{y}\|_{\mathbf{L}^{s}(\Omega)}=\left(\int_{\Omega}|\nabla \mathbf{y}|^{s} d x\right)^{\frac{1}{s}}=\left(\int_{\Omega}\left[\left|\nabla y_{1}\right|^{2}+\left|\nabla y_{2}\right|^{2}\right]^{\frac{s}{2}} d x\right)^{\frac{1}{s}} .
$$

Copyright $@$ by SIAM. Unauthorized reproduction of this article is prohibited. 
We also consider the spaces

$$
\begin{aligned}
& \mathbf{H}=\text { closure of }\left\{\boldsymbol{\phi} \in \mathbf{C}_{0}^{\infty}(\Omega): \operatorname{div} \boldsymbol{\phi}=0\right\} \text { in } \mathbf{L}^{2}(\Omega), \\
& \mathbf{W}_{s}(\Omega)=\left\{\mathbf{y} \in \mathbf{W}_{0}^{1, s}(\Omega): \operatorname{div} \mathbf{y}=0\right\} .
\end{aligned}
$$

For $s=2$ we set $\mathbf{H}_{0}^{1}(\Omega)=\mathbf{W}_{0}^{1,2}(\Omega)$ and $\mathbf{V}=\mathbf{W}_{2}(\Omega)$.

We also define the following spaces

$$
\begin{aligned}
& \mathbf{W}(0, T)=\left\{\mathbf{y} \in L^{2}(I ; \mathbf{V}): \frac{\partial \mathbf{y}}{\partial t} \in L^{2}\left(I ; \mathbf{V}^{\prime}\right)\right\} \\
& \mathbf{W}_{r, s}(0, T)=\left\{\mathbf{y} \in L^{r}\left(I ; \mathbf{W}_{s}(\Omega)\right): \frac{\partial \mathbf{y}}{\partial t} \in L^{r}\left(I ; \mathbf{W}_{s^{\prime}}(\Omega)^{\prime}\right)\right\} \\
& \mathbf{V}^{2,1}(0, T)=\left\{\mathbf{y} \in L^{2}\left(I ; \mathbf{H}^{2}(\Omega) \cap \mathbf{V}\right): \frac{\partial \mathbf{y}}{\partial t} \in L^{2}(I ; \mathbf{H})\right\}
\end{aligned}
$$

with $r, s \in(1, \infty)$, endowed with the norms

$$
\begin{aligned}
& \|\mathbf{y}\|_{\mathbf{W}(0, T)}=\|\mathbf{y}\|_{L^{2}\left(I ; \mathbf{H}_{0}^{1}(\Omega)\right)}+\left\|\frac{\partial \mathbf{y}}{\partial t}\right\|_{L^{2}\left(I ; \mathbf{V}^{\prime}\right)}, \\
& \|\mathbf{y}\|_{\mathbf{W}_{r, s}(0, T)}=\|\mathbf{y}\|_{L^{r}\left(I ; \mathbf{W}_{0}^{1, s}(\Omega)\right)}+\left\|\frac{\partial \mathbf{y}}{\partial t}\right\|_{L^{r}\left(I ; \mathbf{W}_{s^{\prime}}(\Omega)^{\prime}\right)}, \\
& \|\mathbf{y}\|_{\mathbf{V}^{2,1}(0, T)}=\|\mathbf{y}\|_{L^{2}\left(I ; \mathbf{H}^{2}(\Omega)\right)}+\left\|\frac{\partial \mathbf{y}}{\partial t}\right\|_{L^{2}(I ; \mathbf{H})} .
\end{aligned}
$$

Obviously these are reflexive Banach spaces, and $\mathbf{W}(0, T)=\mathbf{W}_{r, s}(0, T)$ if $r=s=2$. Moreover, $\mathbf{W}(0, T)$ and $\mathbf{V}^{2,1}(0, T)$ are Hilbert spaces.

Now we consider the interpolation space $\mathbf{B}_{s, r}(\Omega)=\left(\mathbf{W}_{s^{\prime}}(\Omega)^{\prime}, \mathbf{W}_{s}(\Omega)\right)_{1-1 / r, r}$. From [2, Chapter III/4.10.2] we know that $\mathbf{W}_{r, s}(0, T) \subset C\left([0, T] ; \mathbf{B}_{s, r}(\Omega)\right)$, and the trace mapping $\mathbf{y} \in \mathbf{W}_{r, s}(0, T) \rightarrow \mathbf{y}(0) \in \mathbf{B}_{s, r}(\Omega)$ is surjective. If $r=s=2$, then it is known that $\mathbf{B}_{2,2}(\Omega)=\left(\mathbf{V}^{\prime}, \mathbf{V}\right)_{\frac{1}{2}, 2}=\mathbf{H}$. Hence, the embedding $\mathbf{W}(0, T) \subset$ $C([0, T] ; \mathbf{H})$ holds; see [24, Page 22, Proposition I-2.1] and [29, Page 143, Remark 3].

2. Analysis of the state equation. The aim of this section is to study the wellposedness and differentiability of the mapping control-to-state. The results presented in this section are based on the analysis carried out in [15].

Let us consider the Banach space $\mathbf{Y}_{0}=\mathbf{H}+\mathbf{B}_{p, q}(\Omega)$ with the norm

$$
\left\|\mathbf{y}_{0}\right\|_{\mathbf{Y}_{0}}=\inf _{\mathbf{y}=\mathbf{y}_{1}+\mathbf{y}_{2}}\left\|\mathbf{y}_{1}\right\|_{\mathbf{L}^{2}(\Omega)}+\left\|\mathbf{y}_{2}\right\|_{\mathbf{B}_{p, q}(\Omega)} .
$$

It will be assumed that the initial state $\mathbf{y}_{0}$ in (1.1) is an element of $\mathbf{Y}_{0}$. Now we introduce the following spaces:

$$
\begin{aligned}
& \mathbf{Y}=\left[L^{2}(I ; \mathbf{V}) \cap L^{\infty}(I ; \mathbf{H})\right]+L^{q}\left(I ; \mathbf{W}_{p}(\Omega)\right), \\
& \mathcal{Y}=\mathbf{W}(0, T)+\mathbf{W}_{q, p}(0, T) .
\end{aligned}
$$

They are Banach spaces with the norms

$$
\begin{aligned}
& \|\mathbf{y}\|_{Y}=\inf _{\mathbf{y}=\mathbf{y}_{1}+\mathbf{y}_{2}}\left\|\mathbf{y}_{1}\right\|_{L^{2}\left(I ; \mathbf{H}_{0}^{1}(\Omega)\right)}+\left\|\mathbf{y}_{1}\right\|_{L^{\infty}\left(I ; \mathbf{L}^{2}(\Omega)\right)}+\left\|\mathbf{y}_{2}\right\|_{L^{q}\left(I ; \mathbf{W}_{0}^{1, p}(\Omega)\right)}, \\
& \|\mathbf{y}\|_{\mathcal{Y}}=\inf _{\mathbf{y}=\mathbf{y}_{1}+\mathbf{y}_{2}}\left\|\mathbf{y}_{1}\right\|_{\mathbf{W}(0, T)}+\left\|\mathbf{y}_{2}\right\|_{\mathbf{W}_{q, p}(0, T)} .
\end{aligned}
$$

Note that $\mathcal{Y} \subset \mathbf{Y}$. Moreover, since $\mathbf{W}(0, T)$ and $\mathbf{W}_{q, p}(0, T)$ are reflexive spaces, then $\mathcal{Y}$ is reflexive as well. The solution of (1.1) will be found in $\mathcal{Y}$. 
Definition 2.1. Given $\mathbf{f}_{0} \in L^{q}\left(I, \mathbf{W}^{-1, p}(\Omega)\right), \mathbf{u} \in L^{q}(I ; \mathbf{M}(\omega))$, and $\mathbf{y}_{0} \in \mathbf{Y}_{0}$, we say that $\mathbf{y} \in \mathcal{Y}$ is a solution of (1.1) if

$$
\left\{\begin{array}{l}
\frac{d}{d t}\langle\mathbf{y}(t), \boldsymbol{\psi}\rangle_{\left.\mathbf{W}_{p^{\prime}}(\Omega)\right)^{\prime}, \mathbf{W}_{p^{\prime}}(\Omega)}+a(\mathbf{y}(t), \boldsymbol{\psi})+b(\mathbf{y}(t), \mathbf{y}(t), \boldsymbol{\psi}) \\
=\left\langle\mathbf{f}_{0}(t), \boldsymbol{\psi}\right\rangle_{\mathbf{W}^{-1, p}(\Omega), \mathbf{W}_{0}^{1, p^{\prime}}(\Omega)}+\langle\mathbf{u}(t), \boldsymbol{\psi}\rangle_{\mathbf{M}(\omega), \mathbf{C}_{0}(\omega)} \text { in }(0, T) \quad \forall \boldsymbol{\psi} \in \mathbf{W}_{p^{\prime}}(\Omega), \\
\mathbf{y}(0)=\mathbf{y}_{0},
\end{array}\right.
$$

where the system of differential equations is satisfied in the distribution sense and

$$
\begin{aligned}
& a(\mathbf{y}(t), \boldsymbol{\psi})=\nu \int_{\Omega} \nabla \mathbf{y}(x, t): \nabla \boldsymbol{\psi}(x) d x=\nu \sum_{i=1}^{2} \int_{\Omega} \nabla y_{i}(x, t) \nabla \psi_{i}(x) d x, \\
& b(\mathbf{y}(t), \mathbf{y}(t), \boldsymbol{\psi})=\int_{\Omega}[\mathbf{y}(t) \cdot \nabla] \mathbf{y}(t) \cdot \boldsymbol{\psi} d x .
\end{aligned}
$$

$A$ distribution $\mathfrak{p}$ in $Q$ is called an associated pressure if the equation

$$
\frac{\partial \mathbf{y}}{\partial t}-\nu \Delta \mathbf{y}+(\mathbf{y} \cdot \nabla) \mathbf{y}+\nabla \mathfrak{p}=\mathbf{f}_{0}+\chi_{\omega} \mathbf{u} \text { in } Q
$$

is satisfied in the distribution sense. Then, $(\mathbf{y}, \mathfrak{p})$ is called a solution of (1.1).

Given $\mathbf{y}$ satisfying (2.1), the pressure $\mathfrak{p}$ is obtained by using De Rham's theorem; see [27, Lemma IV-1.4.1]. As pointed out in section 1, the embeddings $\mathbf{W}(0, T) \subset$ $C([0, T] ; \mathbf{H})$ and $\mathbf{W}_{q, p}(0, T) \subset C\left([0, T] ; \mathbf{B}_{p, q}(\Omega)\right)$ hold. Hence, $\mathcal{Y} \subset C\left([0, T] ; \mathbf{Y}_{0}\right)$ and, consequently, the initial condition $\mathbf{y}(0)=\mathbf{y}_{0}$ with $\mathbf{y}_{0} \in \mathbf{Y}_{0}$ makes sense.

The next theorem establishes the well-posedness of the state equation (1.1). It is an immediate consequence of [15, Theorems 2.2 and 2.9].

Theorem 2.2. Suppose that $\left(\mathbf{f}_{0}, \mathbf{y}_{0}\right) \in L^{q}\left(I, \mathbf{W}^{-1, p}(\Omega)\right) \times \mathbf{Y}_{0}$ and that (1.2) holds. Then, system $(2.1)$ has a unique solution $(\mathbf{y}, \mathfrak{p}) \in \mathcal{Y} \times W^{-1, q}\left(I ; L^{p}(\Omega) / \mathbb{R}\right)$ for every $\mathbf{u} \in L^{q}(I ; \mathbf{M}(\omega))$. Furthermore, there exists a nondecreasing function $\eta_{p, q}:[0, \infty) \longrightarrow$ $[0, \infty)$ with $\eta_{p, q}(0)=0$ such that

$$
\|\mathbf{y}\|_{\mathcal{Y}} \leq \eta_{p, q}\left(\left\|\mathbf{f}_{0}\right\|_{L^{q}\left(I ; \mathbf{W}_{p^{\prime}}(\Omega)^{\prime}\right)}+\|\mathbf{u}\|_{L^{q}\left(I ; \mathbf{W}^{-1, p}(\Omega)\right)}+\left\|\mathbf{y}_{0}\right\|_{\mathbf{Y}_{0}}\right)
$$

In addition, if $q \geq 8$ and $\mathbf{y}_{0} \in \mathbf{B}_{2,4}(\Omega)+\mathbf{B}_{p, q}(\Omega)$, then $\mathbf{y}$ belongs to $L^{q}\left(I ; \mathbf{L}^{4}(\Omega)\right)$.

Now, we introduce the mapping $G: L^{q}(I ; \mathbf{M}(\omega)) \longrightarrow \mathcal{Y}$ associating to each control $\mathbf{u} \in L^{q}(I ; \mathbf{M}(\omega))$ the solution $\mathbf{y}_{\mathbf{u}} \in \mathcal{Y}$ of (1.1). Then we have the following differentiability result.

Theorem 2.3. $G$ is of class $C^{\infty}$. Further, given $\mathbf{u}, \mathbf{v}, \mathbf{v}_{1}, \mathbf{v}_{2} \in L^{q}(I ; \mathbf{M}(\omega))$ we have that $\mathbf{z}_{\mathbf{v}}=G^{\prime}(\mathbf{u}) \mathbf{v}$ and $\mathbf{z}_{\mathbf{v}_{1}, \mathbf{v}_{2}}=G^{\prime \prime}(\mathbf{u})\left(\mathbf{v}_{1}, \mathbf{v}_{2}\right)$ are the unique solutions in $\mathcal{Y}$ of the Oseen systems

$$
\left\{\begin{array}{l}
\frac{\partial \mathbf{z}}{\partial t}-\nu \Delta \mathbf{z}+\left(\mathbf{y}_{\mathbf{u}} \cdot \nabla\right) \mathbf{z}+(\mathbf{z} \cdot \nabla) \mathbf{y}_{\mathbf{u}}+\nabla \mathfrak{q}=\chi_{\omega} \mathbf{v} \quad \text { in } Q \\
\operatorname{div} \mathbf{z}=0 \quad \text { in } Q, \mathbf{z}=0 \quad \text { on } \Sigma, \mathbf{z}(0)=0 \text { in } \Omega
\end{array}\right.
$$

and

$$
\left\{\begin{array}{l}
\frac{\partial \mathbf{z}}{\partial t}-\nu \Delta \mathbf{z}+\left(\mathbf{y}_{\mathbf{u}} \cdot \nabla\right) \mathbf{z}+(\mathbf{z} \cdot \nabla) \mathbf{y}_{\mathbf{u}}+\nabla \mathfrak{q}=-\left(\mathbf{z}_{\mathbf{v}_{2}} \cdot \nabla\right) \mathbf{z}_{\mathbf{v}_{1}}-\left(\mathbf{z}_{\mathbf{v}_{1}} \cdot \nabla\right) \mathbf{z}_{\mathbf{v}_{2}} \text { in } Q, \\
\operatorname{div} \mathbf{z}=0 \text { in } Q, \mathbf{z}=0 \text { on } \Sigma, \mathbf{z}(0)=0 \text { in } \Omega,
\end{array}\right.
$$

respectively, where $\mathbf{y}_{\mathbf{u}}=G(\mathbf{u})$ and $\mathbf{z}_{\mathbf{v}_{i}}=G^{\prime}(\mathbf{u}) \mathbf{v}_{i}$ for $i=1,2$. 
Proof. Let $G_{0}: L^{q}\left(I ; \mathbf{W}^{-1, p}(\Omega)\right) \longrightarrow \mathcal{Y}$ be defined by $G_{0}(\mathbf{f})=\mathbf{y}_{\mathbf{f}}$ with $\mathbf{y}_{\mathbf{f}}$ the solution of the system

$$
\left\{\begin{array}{l}
\frac{\partial \mathbf{y}}{\partial t}-\nu \Delta \mathbf{y}+(\mathbf{y} \cdot \nabla) \mathbf{y}+\nabla \mathfrak{p}=\mathbf{f} \text { in } Q=\Omega \times I \\
\operatorname{div} \mathbf{y}=0 \text { in } Q, \mathbf{y}=0 \text { on } \Sigma=\Gamma \times I, \mathbf{y}(0)=\mathbf{y}_{0} \text { in } \Omega
\end{array}\right.
$$

Then, we have that $G(\mathbf{u})=\left(G_{0} \circ B\right)(\mathbf{u})$ with $B: L^{q}(I ; \mathbf{M}(\omega)) \longrightarrow L^{q}\left(I ; \mathbf{W}^{-1, p}(\Omega)\right)$ given by $B \mathbf{u}=\mathbf{f}_{0}+\chi_{\omega} \mathbf{u}$. The statement of the theorem is a straightforward consequence of the chain rule and [15, Theorerm 5.1].

We finish this section proving the a continuity result for $G$.

TheOrem 2.4. Let $\left\{\mathbf{u}_{k}\right\}_{k=1}^{\infty} \subset L^{q}(I ; \mathbf{M}(\omega))$ be a sequence such that $\mathbf{u}_{k} \stackrel{*}{\rightarrow} \mathbf{u}$ in $L^{q}(I ; \mathbf{M}(\omega))$; then $\mathbf{y}_{\mathbf{u}_{k}} \rightarrow \mathbf{y}_{\mathbf{u}}$ in $\mathcal{Y}$ and $\mathbf{y}_{\mathbf{u}_{k}} \rightarrow \mathbf{y}_{\mathbf{u}}$ in $L^{2}\left(I ; \mathbf{H}_{2 p}\right)$, where $\mathbf{H}_{2 p}=$ $\mathbf{H} \cap \mathbf{L}^{2 p}(\Omega)$.

Proof. The boundedness of $\left\{\mathbf{u}_{k}\right\}_{k=1}^{\infty}$ in $L^{q}(I ; \mathbf{M}(\omega))$ along with the estimate (2.2) implies the boundedness of $\left\{\mathbf{y}_{\mathbf{u}_{k}}\right\}_{k=1}^{\infty}$ in $\mathcal{Y}$. Since $\mathcal{Y}$ is reflexive, there exists a subsequence, denoted in the same way, such that $\mathbf{y}_{\mathbf{u}_{k}} \rightarrow \mathbf{y}$ in $\mathcal{Y}$. Now, we pass to the limit in (2.1) satisfied by every pair $\left(\mathbf{y}_{\mathbf{u}_{k}}, \mathbf{u}_{k}\right)$. In this process, the only difficulty is found in the nonlinear term $b\left(\mathbf{y}_{\mathbf{u}_{k}}, \mathbf{y}_{\mathbf{u}_{k}}, \boldsymbol{\psi}\right)$. To deal with it we use a compact embedding. Using the Sobolev embeddings $\mathbf{V} \subset \mathbf{H}_{2 p} \subset \mathbf{V}^{*}$ and $\mathbf{W}_{p}(\Omega) \subset \mathbf{H}_{2 p} \subset \mathbf{W}_{p^{\prime}}(\Omega)^{*}$, which are compact, we have the compactness of the embeddings $\mathbf{W}(0, T) \subset L^{2}\left(I ; \mathbf{H}_{2 p}\right)$ and $\mathbf{W}_{q, p}(0, T) \subset L^{q}\left(I ; \mathbf{H}_{2 p}\right)$; see [28, Theorem III-2.1]. Since $q>4$, we get that the embedding $\mathcal{Y} \subset L^{2}\left(I ; \mathbf{H}_{2 p}\right)$ is compact. Hence, we deduce that $\mathbf{y}_{\mathbf{u}_{k}} \rightarrow \mathbf{y}$ strongly in $L^{2}\left(I ; \mathbf{H}_{2 p}\right)$. Finally, given $\boldsymbol{\psi} \in \mathbf{W}_{p^{\prime}}(\Omega)$ and using the antisymmetric property of $b$ we get

$$
b\left(\mathbf{y}_{\mathbf{u}_{k}}, \mathbf{y}_{\mathbf{u}_{k}}, \boldsymbol{\psi}\right)=-b\left(\mathbf{y}_{\mathbf{u}_{k}}, \boldsymbol{\psi}, \mathbf{y}_{\mathbf{u}_{k}}\right) \rightarrow-b(\mathbf{y}, \boldsymbol{\psi}, \mathbf{y})=b(\mathbf{y}, \mathbf{y}, \boldsymbol{\psi}) \text { strongly in } L^{1}(I) .
$$

Therefore, $\mathbf{y}$ satisfies (2.1) and, hence, $\mathbf{y}=\mathbf{y}_{\mathbf{u}}$. Since every convergent subsequence of $\left\{\mathbf{y}_{\mathbf{u}_{k}}\right\}_{k=1}^{\infty}$ converges to the same limit $\mathbf{y}_{\mathbf{u}}$, we conclude that the whole sequence converges as claimed in the theorem to $\mathbf{y}_{\mathbf{u}}$.

3. Existence of solutions of (P) and first-order optimality conditions. We start this section by proving the existence of solutions for the control problem (P). Then, we show the differentiability of the cost functional and deduce the firstorder necessary optimality conditions. From these conditions we infer the sparsity properties of the stationary controls.

Theorem 3.1. There exists at least one solution $\overline{\mathbf{u}}$ of $(\mathrm{P})$.

Proof. First, we observe that $\mathbf{U}_{\text {ad }}$ is the closed ball of $L^{\infty}(I ; \mathbf{M}(\omega))$ centered at $\mathbf{0}$ and radius $\gamma$. Moreover, $L^{1}\left(I ; \mathbf{C}_{0}(\omega)\right)$ is a separable Banach space and $L^{\infty}(I ; \mathbf{M}(\omega))=$ $L^{1}\left(I ; \mathbf{C}_{0}(\omega)\right)^{*}$. Hence, given a minimizing sequence $\left\{\mathbf{u}_{k}\right\}_{k=1}^{\infty}$ for $(\mathrm{P})$, there exists a subsequence, denoted in the same way, such that $\mathbf{u}_{k} \stackrel{*}{\rightarrow} \overline{\mathbf{u}}$ in $L^{\infty}(I ; \mathbf{M}(\omega))$. Then, Theorem 2.4 implies that $\mathbf{y}_{\mathbf{u}_{k}} \rightarrow \mathbf{y}_{\overline{\mathbf{u}}}$ in $L^{2}(Q)$. Therefore, $J\left(\mathbf{u}_{k}\right) \rightarrow J(\overline{\mathbf{u}})=\inf (\mathrm{P})$ holds. Thus, $\overline{\mathbf{u}}$ is a solution of $(\mathrm{P})$.

Before stating the optimality conditions satisfied by a solution of $(\mathrm{P})$, we analyze the differentiability of the cost functional.

Theorem 3.2. The cost functional $J: L^{q}(I ; \mathbf{M}(\omega)) \longrightarrow \mathbb{R}$ is of class $C^{\infty}$ and the following identities hold 


$$
\begin{aligned}
& J^{\prime}(\mathbf{u}) \mathbf{v}=\int_{0}^{T}\left\langle\mathbf{v}(t), \boldsymbol{\varphi}_{\mathbf{u}}(t)\right\rangle_{\mathbf{M}(\omega), \mathbf{C}_{0}(\omega)} d t \\
& J^{\prime \prime}(\mathbf{u}) \mathbf{v}^{2}=\int_{Q}\left\{\left|\mathbf{z}_{\mathbf{v}}\right|^{2}+2\left(\mathbf{z}_{\mathbf{v}} \cdot \nabla\right) \boldsymbol{\varphi}_{\mathbf{u}} \mathbf{z}_{\mathbf{v}}\right\} d x d t
\end{aligned}
$$

for all $\mathbf{v} \in L^{q}(I ; \mathbf{M}(\omega))$, where $\mathbf{z}_{\mathbf{v}}=G^{\prime}(\mathbf{u}) \mathbf{v}$ and $\boldsymbol{\varphi}_{\mathbf{u}} \in \mathbf{V}^{2,1}(0, T)$ is the adjoint state, the unique solution along with the pressure $\pi_{\mathbf{u}}$ of

$$
\left\{\begin{array}{l}
-\frac{\partial \varphi}{\partial t}-\nu \Delta \boldsymbol{\varphi}-\left(\mathbf{y}_{\mathbf{u}} \cdot \nabla\right) \boldsymbol{\varphi}-(\nabla \boldsymbol{\varphi})^{T} \mathbf{y}_{\mathbf{u}}+\nabla \pi=\mathbf{y}_{\mathbf{u}}-\mathbf{y}_{d} \text { in } Q \\
\operatorname{div} \boldsymbol{\varphi}=0 \text { in } Q, \boldsymbol{\varphi}=0 \text { on } \Sigma, \boldsymbol{\varphi}(T)=0 \text { in } \Omega
\end{array}\right.
$$

Proof. The differentiability of $J$ is a consequence of the chain rule and Theorem 2.3. The expressions (3.1) and (3.2) follow from (2.3), (2.4), and (3.3). We only have to prove that (3.3) has a unique solution that belongs to $\mathbf{V}^{2,1}(0, T)$. To this end, let us consider the classical operator associated with the Stokes system $A: \mathbf{V} \longrightarrow \mathbf{V}^{\prime}$ given by $\langle A \boldsymbol{\psi}, \boldsymbol{\phi}\rangle_{\mathbf{V}^{\prime}, \mathbf{V}}=a(\boldsymbol{\psi}, \boldsymbol{\phi}) \forall \boldsymbol{\psi}, \boldsymbol{\phi} \in \mathbf{V}$. As usual, we take a basis $\left\{\boldsymbol{\psi}_{j}\right\}_{j=1}^{\infty}$ of $\mathbf{V}$ formed by eigenfunctions of $A$ : $A \boldsymbol{\psi}_{j}=\lambda_{j} \boldsymbol{\psi}_{j}$ with $\left\{\lambda_{j}\right\}_{j=1}^{\infty} \subset(0, \infty), j \geq 1$. We assume that $\left\{\boldsymbol{\psi}_{j}\right\}_{j=1}^{\infty}$ is orthonormal for the Hilbert product in $\mathbf{H}:\left(\psi_{i}, \boldsymbol{\psi}_{j}\right)_{\mathbf{L}^{2}(\Omega)}=\delta_{i j}$. Let us denote by $\mathbf{V}_{k}$ the subspace generated by $\left\{\boldsymbol{\psi}_{1}, \ldots, \boldsymbol{\psi}_{k}\right\}$. Following the classical Faedo-Galerkin approach, we discretize (3.3)

$$
\left\{\begin{array}{l}
-\frac{d}{d t}\left(\boldsymbol{\varphi}_{k}(t), \boldsymbol{\psi}_{j}\right)_{\mathbf{L}^{2}(\Omega)}+a\left(\boldsymbol{\varphi}_{k}(t), \boldsymbol{\psi}_{j}\right)-b\left(\mathbf{y}_{\mathbf{u}}(t), \boldsymbol{\varphi}_{k}(t), \boldsymbol{\psi}_{j}\right) \\
-b\left(\boldsymbol{\psi}_{j}, \boldsymbol{\varphi}_{k}(t), \mathbf{y}_{\mathbf{u}}(t)\right)=\left(\mathbf{y}_{\mathbf{u}}(t)-\mathbf{y}_{d}(t), \boldsymbol{\psi}_{j}\right)_{\mathbf{L}^{2}(\Omega)} \text { in }(0, T), 1 \leq j \leq k, \\
\boldsymbol{\varphi}_{k}(T)=0
\end{array}\right.
$$

where $\boldsymbol{\varphi}_{k}(t)=\sum_{j=1}^{k} g_{k, j}(t) \boldsymbol{\psi}_{j}$. Arguing analogously as in [15, Proof of Proposition 2.7], we infer the existence and uniqueness of a solution $\varphi_{k}$ satisfying the estimate

$$
\left\|\boldsymbol{\varphi}_{k}\right\|_{\mathbf{W}(0, T)} \leq \eta_{0}\left(\left\|\mathbf{y}_{\mathbf{u}}\right\|_{\mathbf{Y}}\right)\left\|\mathbf{y}_{\mathbf{u}}-\mathbf{y}_{d}\right\|_{\mathbf{L}^{2}(Q)} \quad \forall k
$$

where $\eta_{0}:[0, \infty) \longrightarrow[0, \infty)$ is a nondecreasing function vanishing at 0 . Moreover, as in [15], we can prove that $\left\{\boldsymbol{\varphi}_{k}\right\}_{k=1}^{\infty}$ converges weakly in $\mathbf{W}(0, T)$ to the unique solution $\varphi_{\mathbf{u}}$ of (3.3). Moreover, $\boldsymbol{\varphi}_{\mathbf{u}}$ also satisfies the estimate (3.5). It remains to prove the $\mathbf{V}^{2,1}(0, T)$ regularity. To this end, we split the proof into two parts.

I. Estimate of $\left\|\boldsymbol{\varphi}_{k}\right\|_{L^{2}\left(I ; \mathbf{H}^{2}(\Omega) \cap \mathbf{V}\right)}$. First, we observe that

$$
A \boldsymbol{\varphi}_{k}=\sum_{j=1}^{k} g_{k, j} A \boldsymbol{\psi}_{j}=\sum_{j=1}^{k} \lambda_{j} g_{k, j} \boldsymbol{\psi}_{j}
$$

Multiplying (3.4) by $\lambda_{j} g_{k, j}(t)$ and taking the sum from $j=1$ to $k$ we infer

$$
\begin{aligned}
& -\frac{d}{d t}\left(\boldsymbol{\varphi}_{k}(t), A \boldsymbol{\varphi}_{k}(t)\right)_{\mathbf{L}^{2}(\Omega)}+a\left(\boldsymbol{\varphi}_{k}(t), A \boldsymbol{\varphi}_{k}(t)\right)-b\left(\mathbf{y}_{\mathbf{u}}(t), \boldsymbol{\varphi}_{k}(t), A \boldsymbol{\varphi}_{k}(t)\right) \\
& -b\left(A \boldsymbol{\varphi}_{k}(t), \boldsymbol{\varphi}_{k}(t), \mathbf{y}_{\mathbf{u}}(t)\right)=\left(\mathbf{y}_{\mathbf{u}}(t)-\mathbf{y}_{d}(t), A \boldsymbol{\varphi}_{k}(t)\right)_{\mathbf{L}^{2}(\Omega)} .
\end{aligned}
$$

Using the identities established in [5, page 372], the above identity yields

$$
\begin{aligned}
- & \left.\frac{1}{2} \frac{d}{d t}\left\|\boldsymbol{\varphi}_{k}(t)\right\|_{\mathbf{H}_{0}^{1}(\Omega)}^{2}+\| A \boldsymbol{\varphi}_{k}(t)\right) \|_{\mathbf{L}^{2}(\Omega)}^{2}=\left(\mathbf{y}_{\mathbf{u}}(t)-\mathbf{y}_{d}(t), A \boldsymbol{\varphi}_{k}(t)\right)_{\mathbf{L}^{2}(\Omega)} \\
& +b\left(\mathbf{y}_{\mathbf{u}}(t), \boldsymbol{\varphi}_{k}(t), A \boldsymbol{\varphi}_{k}(t)\right)+b\left(A \boldsymbol{\varphi}_{k}(t), \boldsymbol{\varphi}_{k}(t), \mathbf{y}_{\mathbf{u}}(t)\right) .
\end{aligned}
$$

Copyright $\odot$ by SIAM. Unauthorized reproduction of this article is prohibited. 
Now, we estimate the right-hand side of this identity. First we get

$$
\begin{aligned}
\left|b\left(\mathbf{y}_{\mathbf{u}}(t), \boldsymbol{\varphi}_{k}(t), A \boldsymbol{\varphi}_{k}(t)\right)\right| & \left.\leq\left\|\mathbf{y}_{\mathbf{u}}(t)\right\|_{\mathbf{L}^{4}(\Omega)}\left\|\nabla \boldsymbol{\varphi}_{k}(t)\right\|_{\mathbf{L}^{4}(\Omega)} \| A \boldsymbol{\varphi}_{k}(t)\right) \|_{\mathbf{L}^{2}(\Omega)} \\
& \left.\leq C_{1}\left\|\mathbf{y}_{\mathbf{u}}(t)\right\|_{\mathbf{L}^{4}(\Omega)}\left\|\nabla \boldsymbol{\varphi}_{k}(t)\right\|_{\mathbf{L}^{2}(\Omega)}^{1 / 2}\left\|\boldsymbol{\varphi}_{k}(t)\right\|_{\mathbf{H}^{2}(\Omega)}^{1 / 2} \| A \boldsymbol{\varphi}_{k}(t)\right) \|_{\mathbf{L}^{2}(\Omega)} \\
& \leq C_{2}\left\|\mathbf{y}_{\mathbf{u}}(t)\right\|_{\mathbf{L}^{4}(\Omega)}\left\|\boldsymbol{\varphi}_{k}(t)\right\|_{\mathbf{H}_{0}^{1}(\Omega)}^{1 / 2}\left\|A \boldsymbol{\varphi}_{k}(t)\right\|_{\mathbf{L}^{2}(\Omega)}^{3 / 2} \\
& \leq \frac{C_{2}^{4}}{4}\left(\frac{9}{2}\right)^{3}\left\|\mathbf{y}_{\mathbf{u}}(t)\right\|_{\mathbf{L}^{4}(\Omega)}^{4}\left\|\boldsymbol{\varphi}_{k}(t)\right\|_{\mathbf{H}_{0}^{1}(\Omega)}^{2}+\frac{1}{6}\left\|A \boldsymbol{\varphi}_{k}(t)\right\|_{\mathbf{L}^{2}(\Omega)}^{2} .
\end{aligned}
$$

Above we have used a Gagliardo inequality (see [5, Proposition III.2.35]), the $\mathbf{H}^{2}(\Omega)$ estimates for the solution of the Stokes problem $\|\mathbf{y}\|_{\mathbf{H}^{2}(\Omega)} \leq C\|A \mathbf{y}\|_{\mathbf{L}^{2}(\Omega)}[5$, Theorem IV.5.8], and Young's inequality.

The estimate for $b\left(A \boldsymbol{\varphi}_{k}(t), \boldsymbol{\varphi}_{k}(t), \mathbf{y}_{\mathbf{u}}(t)\right)$ is exactly the same. Therefore, inserting these estimates in (3.6) and using again Young's inequality we get

$$
\begin{aligned}
- & \left.\left.\frac{1}{2} \frac{d}{d t}\left\|\boldsymbol{\varphi}_{k}(t)\right\|_{\mathbf{H}_{0}^{1}(\Omega)}^{2}+\| A \boldsymbol{\varphi}_{k}(t)\right)\left\|_{\mathbf{L}^{2}(\Omega)}^{2} \leq\right\| \mathbf{y}_{\mathbf{u}}(t)-\mathbf{y}_{d}\left\|_{\mathbf{L}^{2}(\Omega)}\right\| A \boldsymbol{\varphi}_{k}(t)\right) \|_{\mathbf{L}^{2}(\Omega)} \\
& +C_{3}\left\|\mathbf{y}_{\mathbf{u}}(t)\right\|_{\mathbf{L}^{4}(\Omega)}^{4}\left\|\boldsymbol{\varphi}_{k}(t)\right\|_{\mathbf{H}_{0}^{1}(\Omega)}^{2}+\frac{1}{3}\left\|A \boldsymbol{\varphi}_{k}(t)\right\|_{\mathbf{L}^{2}(\Omega)}^{2} \\
& \leq \frac{3}{2}\left\|\mathbf{y}_{\mathbf{u}}(t)-\mathbf{y}_{d}\right\|_{\mathbf{L}^{2}(\Omega)}^{2}+C_{3}\left\|\mathbf{y}_{\mathbf{u}}(t)\right\|_{\mathbf{L}^{4}(\Omega)}^{4}\left\|\boldsymbol{\varphi}_{k}(t)\right\|_{\mathbf{H}_{0}^{1}(\Omega)}^{2}+\frac{1}{2}\left\|A \boldsymbol{\varphi}_{k}(t)\right\|_{\mathbf{L}^{2}(\Omega)}^{2},
\end{aligned}
$$

which implies

$$
\begin{aligned}
& \left.-\frac{d}{d t}\left\|\boldsymbol{\varphi}_{k}(t)\right\|_{\mathbf{H}_{0}^{1}(\Omega)}^{2}+\| A \boldsymbol{\varphi}_{k}(t)\right) \|_{\mathbf{L}^{2}(\Omega)}^{2} \\
& \quad \leq 3\left\|\mathbf{y}_{\mathbf{u}}(t)-\mathbf{y}_{d}\right\|_{\mathbf{L}^{2}(\Omega)}^{2}+2 C_{3}\left\|\mathbf{y}_{\mathbf{u}}(t)\right\|_{\mathbf{L}^{4}(\Omega)}^{4}\left\|\boldsymbol{\varphi}_{k}(t)\right\|_{\mathbf{H}_{0}^{1}(\Omega)}^{2} .
\end{aligned}
$$

Let us prove that $\mathbf{y}_{\mathbf{u}} \in L^{4}\left(I ; \mathbf{L}^{4}(\Omega)\right)$. Since $\mathbf{y}_{\mathbf{u}} \in \mathcal{Y}$, we can write it in the form $\mathbf{y}_{\mathbf{u}}=\mathbf{y}_{1}+\mathbf{y}_{2}$ with $\mathbf{y}_{1} \in \mathbf{W}(0, T)$ and $\mathbf{y}_{2} \in \mathbf{W}_{q, p}(0, T)$. Using again a Gagliardo inequality we obtain

$$
\left\|\mathbf{y}_{1}(t)\right\|_{\mathbf{L}^{4}(\Omega)}^{4} \leq C_{4}\left\|\mathbf{y}_{1}(t)\right\|_{\mathbf{L}^{2}(\Omega)}^{2}\left\|\mathbf{y}_{1}(t)\right\|_{\mathbf{H}_{0}^{1}(\Omega)}^{2} \leq C_{4}\left\|\mathbf{y}_{1}\right\|_{L^{\infty}\left(I ; \mathbf{L}^{2}(\Omega)\right)}^{2}\left\|\mathbf{y}_{1}(t)\right\|_{\mathbf{H}_{0}^{1}(\Omega)}^{2} .
$$

The embeddings $\mathbf{W}(0, T) \subset L^{2}\left(I ; \mathbf{H}_{0}^{1}(\Omega)\right)$ and $\mathbf{W}(0, T) \subset L^{\infty}\left(I ; \mathbf{L}^{2}(\Omega)\right)$ and the above inequality imply $\mathbf{y}_{1} \in L^{4}\left(I ; \mathbf{L}^{4}(\Omega)\right)$. On the other hand, since $\mathbf{W}_{q, p}(0, T) \subset$ $L^{q}\left(I ; \mathbf{W}_{p}(\Omega)\right) \subset L^{4}\left(I ; \mathbf{L}^{4}(\Omega)\right)$, recall $(1.2)$; we infer that $\mathbf{y}_{2} \in L^{4}\left(I ; \mathbf{L}^{4}(\Omega)\right)$. Then, $\mathbf{y}_{\mathbf{u}} \in L^{4}\left(I ; \mathbf{L}^{4}(\Omega)\right)$ holds. Now, integrating $(3.7)$ in $[t, T]$ and using that $\boldsymbol{\varphi}_{k}(T)=0$ it follows

$$
\left\|\boldsymbol{\varphi}_{k}(t)\right\|_{\mathbf{H}_{0}^{1}(\Omega)}^{2} \leq 3\left\|\mathbf{y}_{\mathbf{u}}-\mathbf{y}_{d}\right\|_{\mathbf{L}^{2}(Q)}^{2}+2 C_{3} \int_{t}^{T}\left\|\mathbf{y}_{\mathbf{u}}(s)\right\|_{\mathbf{L}^{4}(\Omega)}^{4}\left\|\boldsymbol{\varphi}_{k}(s)\right\|_{\mathbf{H}_{0}^{1}(\Omega)}^{2} d s \forall t \in I .
$$

Applying Gronwall inequality we infer

$$
\left\|\boldsymbol{\varphi}_{k}\right\|_{L^{\infty}\left(I ; \mathbf{H}_{0}^{1}(\Omega)\right)} \leq \sqrt{3}\left\|\mathbf{y}_{\mathbf{u}}-\mathbf{y}_{d}\right\|_{\mathbf{L}^{2}(Q)} \exp \left(C_{3}\left\|\mathbf{y}_{\mathbf{u}}\right\|_{L^{4}\left(I ; \mathbf{L}^{4}(\Omega)\right)}^{4}\right) \quad \forall k \geq 1 .
$$

Finally, integrating (3.7) in $[0, T]$ and inserting (3.8) we obtain

$$
\begin{aligned}
& \left\|A \boldsymbol{\varphi}_{k}\right\|_{\mathbf{L}^{2}(Q)} \\
& \quad \leq \sqrt{3}\left\|\mathbf{y}_{\mathbf{u}}-\mathbf{y}_{d}\right\|_{\mathbf{L}^{2}(Q)}\left\{1+\sqrt{2 C_{3}}\left\|\mathbf{y}_{\mathbf{u}}\right\|_{L^{4}\left(I ; \mathbf{L}^{4}(\Omega)\right)}^{2} \exp \left(C_{3}\left\|\mathbf{y}_{\mathbf{u}}\right\|_{L^{4}\left(I ; \mathbf{L}^{4}(\Omega)\right)}^{4}\right)\right\} .
\end{aligned}
$$

Copyright $@$ by SIAM. Unauthorized reproduction of this article is prohibited. 
Once again, with $\|\mathbf{y}\|_{\mathbf{H}^{2}(\Omega)} \leq C\|A \mathbf{y}\|_{\mathbf{L}^{2}(\Omega)}[5$, Theorem IV.5.8] we deduce from the above estimate for all $k \geq 1$

$$
\begin{aligned}
& \left\|\boldsymbol{\varphi}_{k}\right\|_{L^{2}\left(I ; \mathbf{H}^{2}(\Omega)\right)} \\
& \quad \leq C\left\|\mathbf{y}_{\mathbf{u}}-\mathbf{y}_{d}\right\|_{\mathbf{L}^{2}(Q)}\left\{1+\hat{C}\left\|\mathbf{y}_{\mathbf{u}}\right\|_{L^{4}\left(I ; \mathbf{L}^{4}(\Omega)\right)}^{2} \exp \left(\hat{C}^{2}\left\|\mathbf{y}_{\mathbf{u}}\right\|_{L^{4}\left(I ; \mathbf{L}^{4}(\Omega)\right)}^{4}\right)\right\} .
\end{aligned}
$$

II. Estimate of $\left\|\boldsymbol{\varphi}_{k}^{\prime}\right\|_{L^{2}(I ; \mathbf{H})}$. Multiplying (3.4) by $-g_{k, j}^{\prime} \boldsymbol{\psi}_{j}$, adding the resulting identities from $j=1$ to $k$, using the orthogonality of $\left\{\boldsymbol{\psi}_{j}\right\}_{j=1}^{\infty}$ in $\mathbf{H}$, and integrating in $[0, T]$ we get

$$
\begin{gathered}
\left\|\boldsymbol{\varphi}_{k}^{\prime}\right\|_{\mathbf{L}^{2}(Q)}^{2}-\frac{1}{2} \int_{0}^{T} \frac{d}{d t} a\left(\boldsymbol{\varphi}_{k}(t), \boldsymbol{\varphi}_{k}(t)\right) d t=-\int_{0}^{T}\left(\mathbf{y}_{\mathbf{u}}(t)-\mathbf{y}_{d}(t), \boldsymbol{\varphi}_{k}^{\prime}(t)\right)_{\mathbf{L}^{2}(\Omega)} d t \\
-\int_{0}^{T} b\left(\mathbf{y}_{\mathbf{u}}(t), \boldsymbol{\varphi}_{k}(t), \boldsymbol{\varphi}_{k}^{\prime}(t)\right) d t-\int_{0}^{T} b\left(\boldsymbol{\varphi}_{k}^{\prime}(t), \boldsymbol{\varphi}_{k}(t), \mathbf{y}_{\mathbf{u}}(t)\right) d t
\end{gathered}
$$

Now, taking into account that $\varphi_{k}(T)=0$ it follows from the above identity

$$
\begin{aligned}
\left\|\boldsymbol{\varphi}_{k}^{\prime}\right\|_{\mathbf{L}^{2}(Q)}^{2} \leq & \left\|\mathbf{y}_{\mathbf{u}}-\mathbf{y}_{d}\right\|_{\mathbf{L}^{2}(Q)}\left\|\boldsymbol{\varphi}_{k}^{\prime}\right\|_{\mathbf{L}^{2}(Q)} \\
& +\mid \int_{0}^{T} b\left(\mathbf{y}_{\mathbf{u}}(t), \boldsymbol{\varphi}_{k}(t), \boldsymbol{\varphi}_{k}^{\prime}(t)\right) d t+\int_{0}^{T} b\left(\boldsymbol{\varphi}_{k}^{\prime}(t), \boldsymbol{\varphi}_{k}(t), \mathbf{y}_{\mathbf{u}}(t)\right) d t .
\end{aligned}
$$

With the Gagliardo and Young inequalities we obtain

$$
\begin{aligned}
\left|b\left(\mathbf{y}_{\mathbf{u}}(t), \boldsymbol{\varphi}_{k}(t), \boldsymbol{\varphi}_{k}^{\prime}(t)\right)\right| & \leq\left\|\mathbf{y}_{\mathbf{u}}\right\|_{\mathbf{L}^{4}(\Omega)}\left\|\nabla \boldsymbol{\varphi}_{k}\right\|_{\mathbf{L}^{4}(\Omega)}\left\|\boldsymbol{\varphi}_{k}^{\prime}\right\|_{\mathbf{L}^{2}(\Omega)} \\
& \left.\leq C_{1}\left\|\mathbf{y}_{\mathbf{u}}(t)\right\|_{\mathbf{L}^{4}(\Omega)}\left\|\nabla \boldsymbol{\varphi}_{k}(t)\right\|_{\mathbf{L}^{2}(\Omega)}^{1 / 2}\left\|\boldsymbol{\varphi}_{k}(t)\right\|_{\mathbf{H}^{2}(\Omega)}^{1 / 2} \| \boldsymbol{\varphi}_{k}^{\prime}(t)\right) \|_{\mathbf{L}^{2}(\Omega)} \\
& \left.\leq \frac{3 C_{1}^{2}}{2}\left\|\mathbf{y}_{\mathbf{u}}(t)\right\|_{\mathbf{L}^{4}(\Omega)}^{2}\left\|\nabla \boldsymbol{\varphi}_{k}(t)\right\|_{\mathbf{L}^{2}(\Omega)}\left\|\boldsymbol{\varphi}_{k}(t)\right\|_{\mathbf{H}^{2}(\Omega)}+\frac{1}{6} \| \boldsymbol{\varphi}_{k}^{\prime}(t)\right) \|_{\mathbf{L}^{2}(\Omega)}^{2}
\end{aligned}
$$

The same estimate is valid for $\left|b\left(\varphi_{k}^{\prime}(t), \boldsymbol{\varphi}_{k}(t), \mathbf{y}_{\mathbf{u}}(t)\right)\right|$. Inserting these estimates in (3.10) and using Schwarz's inequality we find

$$
\begin{aligned}
& \left|\int_{0}^{T} b\left(\mathbf{y}_{\mathbf{u}}(t), \boldsymbol{\varphi}_{k}(t), \boldsymbol{\varphi}_{k}^{\prime}(t)\right) d t+\int_{0}^{T} b\left(\boldsymbol{\varphi}_{k}^{\prime}(t), \boldsymbol{\varphi}_{k}(t), \mathbf{y}_{\mathbf{u}}(t)\right) d t\right| \\
& \left.\quad \leq 3 C_{1}^{2}\left\|\boldsymbol{\varphi}_{k}\right\|_{L^{\infty}\left(I ; \mathbf{H}_{0}^{1}(\Omega)\right)}\left\|\mathbf{y}_{\mathbf{u}}\right\|_{L^{4}\left(I ; \mathbf{L}^{4}(\Omega)\right)}^{2}\left\|\boldsymbol{\varphi}_{k}\right\|_{L^{2}\left(I ; \mathbf{H}^{2}(\Omega)\right)}+\frac{1}{3} \| \boldsymbol{\varphi}_{k}^{\prime}(t)\right) \|_{\mathbf{L}^{2}(\Omega)}^{2} .
\end{aligned}
$$

This estimate, (3.10), and Young's inequality lead to

$$
\begin{aligned}
& \left\|\boldsymbol{\varphi}_{k}^{\prime}\right\|_{\mathbf{L}^{2}(Q)}^{2} \leq \frac{3}{2}\left\|\mathbf{y}_{\mathbf{u}}-\mathbf{y}_{d}\right\|_{\mathbf{L}^{2}(Q)}^{2}+\frac{1}{6}\left\|\boldsymbol{\varphi}_{k}^{\prime}\right\|_{\mathbf{L}^{2}(Q)}^{2} \\
& \left.\quad+3 C_{1}^{2}\left\|\boldsymbol{\varphi}_{k}\right\|_{L^{\infty}\left(I ; \mathbf{H}_{0}^{1}(\Omega)\right)}\left\|\mathbf{y}_{\mathbf{u}}\right\|_{L^{4}\left(I ; \mathbf{L}^{4}(\Omega)\right)}^{2}\left\|\boldsymbol{\varphi}_{k}\right\|_{L^{2}\left(I ; \mathbf{H}^{2}(\Omega)\right)}+\frac{1}{3} \| \boldsymbol{\varphi}_{k}^{\prime}(t)\right) \|_{\mathbf{L}^{2}(\Omega)}^{2},
\end{aligned}
$$

whence

$$
\begin{aligned}
\left\|\boldsymbol{\varphi}_{k}^{\prime}\right\|_{\mathbf{L}^{2}(Q)} \leq & \sqrt{3}\left\|\mathbf{y}_{\mathbf{u}}-\mathbf{y}_{d}\right\|_{\mathbf{L}^{2}(Q)} \\
& +\sqrt{6} C_{1}\left\|\mathbf{y}_{\mathbf{u}}\right\|_{L^{4}\left(I ; \mathbf{L}^{4}(\Omega)\right)}\left\|\boldsymbol{\varphi}_{k}\right\|_{L^{\infty}\left(I ; \mathbf{H}_{0}^{1}(\Omega)\right)}^{1 / 2}\left\|\boldsymbol{\varphi}_{k}\right\|_{\left.L^{2} ; \mathbf{H}^{2}(\Omega)\right)}^{1 / 2}
\end{aligned}
$$

From (3.8) and (3.9) the boundedness of $\left\{\boldsymbol{\varphi}_{k}^{\prime}\right\}_{k=1}^{\infty}$ in $\mathbf{L}^{2}(Q)$ follows. Therefore, $\boldsymbol{\varphi}_{\mathbf{u}}^{\prime} \in$ $\mathbf{L}^{2}(Q)$ holds, and with the first part of the proof we conclude that $\varphi_{\mathbf{u}} \in \mathbf{V}^{2,1}(0, T)$.

Copyright $@$ ㅇ by SIAM. Unauthorized reproduction of this article is prohibited. 
Let us note that the estimates (2.2), (3.5), (3.8), (3.9), and (3.11) yields

$$
\left\|\boldsymbol{\varphi}_{\mathbf{u}}\right\|_{\mathbf{V}^{2,1}(0, T)} \leq \eta\left(\left\|\mathbf{f}_{0}\right\|_{L^{q}\left(I ; \mathbf{W}_{p^{\prime}}(\Omega)^{\prime}\right)}+\|\mathbf{u}\|_{L^{q}(I ; \mathbf{M}(\omega))}+\left\|\mathbf{y}_{0}\right\|_{\mathbf{Y}_{0}}\right)\left\|\mathbf{y}_{\mathbf{u}}-\mathbf{y}_{d}\right\|_{\mathbf{L}^{2}(Q)}
$$

for some nondecreasing monotone function $\eta:[0, \infty) \longrightarrow[0, \infty)$.

Next, we prove the first order necessary optimality conditions. Since $(\mathrm{P})$ is not a convex problem, it is convenient to discuss necessary optimality conditions in the context of local solutions. Here, we say that $\overline{\mathbf{u}}$ is a local solution of $(\mathrm{P})$ if there exists a neighborhood $\mathcal{A}$ of $\overline{\mathbf{u}}$ in $L^{\infty}(I ; \mathbf{M}(\omega))$ such that $J(\overline{\mathbf{u}}) \leq J(\mathbf{u})$ for all $\mathbf{u} \in \mathcal{A}$. If the inequality is strict for all $\mathbf{u} \in \mathcal{A}$ with $\mathbf{u} \neq \overline{\mathbf{u}}$, we say that $\overline{\mathbf{u}}$ is a strict local solution. We will also consider local solutions in the $L^{q}\left(I ; \mathbf{W}^{-1, p}(\Omega)\right)$ topology. Let us observe that the continuous embedding $L^{\infty}(I ; \mathbf{M}(\omega)) \subset L^{q}\left(I ; \mathbf{W}^{-1, p}(\Omega)\right)$ implies that any local solution in the $L^{q}\left(I ; \mathbf{W}^{-1, p}(\Omega)\right)$ topology is also a local solution in the $L^{\infty}(I ; \mathbf{M}(\omega))$ topology.

THEOREM 3.3. Let us assume that $\overline{\mathbf{u}}$ is a local solution of $(\mathrm{P})$ with associated state $\overline{\mathbf{y}}$. Then, there exists a unique element $\bar{\varphi} \in \mathbf{V}^{2,1}(0, T)$ satisfying

$$
\begin{aligned}
& \left\{\begin{array}{l}
-\frac{\partial \bar{\varphi}}{\partial t}-\nu \Delta \bar{\varphi}-(\overline{\mathbf{y}} \cdot \nabla) \bar{\varphi}-(\nabla \bar{\varphi})^{T} \overline{\mathbf{y}}+\nabla \bar{\pi}=\overline{\mathbf{y}}-\mathbf{y}_{d} \text { in } Q, \\
\operatorname{div} \bar{\varphi}=0 \text { in } Q, \bar{\varphi}=0 \text { on } \Sigma, \bar{\varphi}(T)=0 \text { in } \Omega,
\end{array}\right. \\
& \left\{\begin{array}{l}
\text { if } \bar{\varphi}_{i}(t) \not \equiv 0, \text { then }\left\|\bar{u}_{i}(t)\right\|_{M(\omega)}=\gamma \text { and } \\
\operatorname{Supp}\left(\bar{u}_{i}^{+}(t)\right) \subset\left\{x \in \omega: \bar{\varphi}_{i}(x, t)=-\left\|\bar{\varphi}_{i}(t)\right\|_{C_{0}(\omega)}\right\}, \\
\operatorname{Supp}\left(\bar{u}_{i}^{-}(t)\right) \subset\left\{x \in \omega: \bar{\varphi}_{i}(x, t)=+\left\|\bar{\varphi}_{i}(t)\right\|_{C_{0}(\omega)}\right\}
\end{array}\right.
\end{aligned}
$$

for $i=1,2$ and almost every point $t \in I$, where $\bar{u}_{i}(t)=\bar{u}_{i}^{+}(t)-\bar{u}_{i}^{-}(t)$ is the Jordan decomposition of the measure $\bar{u}_{i}(t)$.

Proof. From Theorem 3.2 we know the existence and uniqueness of $\bar{\varphi} \in \mathbf{V}^{2,1}(0, T)$ satisfying (3.13). From the expression for $J^{\prime}$ given in (3.1) and using the convexity of $\mathbf{U}_{\text {ad }}$ we have

$$
0 \leq J^{\prime}(\overline{\mathbf{u}})(\mathbf{u}-\overline{\mathbf{u}})=\int_{0}^{T}\langle\mathbf{u}(t)-\overline{\mathbf{u}}(t), \bar{\varphi}(t)\rangle_{\mathbf{M}(\omega), \mathbf{C}_{0}(\omega)} d t \quad \forall \mathbf{u} \in \mathbf{U}_{\mathrm{ad}}
$$

This is equivalent to

$$
\int_{0}^{T}\left\langle u(t), \bar{\varphi}_{i}(t)\right\rangle_{M(\omega), C_{0}(\omega)} d t \leq-\int_{0}^{T}\left\langle\bar{u}_{i}(t), \bar{\varphi}_{i}(t)\right\rangle_{M(\omega), C_{0}(\omega)} d t, \quad i=1,2
$$

for every $u$ satisfying $\|u\|_{L^{\infty}(I ; M(\omega))} \leq \gamma$.

Since $\bar{\varphi}_{i}: \bar{\Omega} \times I \rightarrow \mathbb{R}$ is a Caratheodory function (continuous with respect to the first variable and measurable with respect to the second), there exists a measurable selection $t \in I \mapsto x_{t} \in \bar{\Omega}$ such that $\left|\bar{\varphi}_{i}\left(x_{t}, t\right)\right|=\left\|\bar{\varphi}_{i}(t)\right\|_{C_{0}(\omega)}$; see [19, Chapter 8, Theorem 1.2]. Now, we define the element $u \in L^{\infty}(I ; M(\omega))$ by $u(t)=\gamma \operatorname{sign}\left(\bar{\varphi}_{i}\left(x_{t}, t\right)\right) \delta_{x_{t}}$. We have to check that $u: I \rightarrow M(\omega)$ is weakly measurable. To this end the only delicate point is the weak measurability of $t \in I \mapsto \delta_{x_{t}} \in M(\omega)$. This follows from the measurability of the mapping $t \mapsto x_{t}$ and the continuity of $x \in \bar{\Omega} \mapsto \delta_{x} \in M(\omega)$ when $M(\omega)$ is endowed with the weak* topology. By definition of $u$, the fact that $\overline{\mathbf{u}} \in \mathbf{U}_{\mathrm{ad}}$, and (3.15) we get 


$$
\begin{aligned}
\gamma \int_{0}^{T}\left\|\bar{\varphi}_{i}(t)\right\|_{C_{0}(\omega)} d t & =\int_{0}^{T}\left\langle u(t), \bar{\varphi}_{i}(t)\right\rangle_{M(\omega), C_{0}(\omega)} d t \\
& \leq-\int_{0}^{T}\left\langle\bar{u}_{i}(t), \bar{\varphi}_{i}(t)\right\rangle_{M(\omega), C_{0}(\omega)} d t \leq \gamma \int_{0}^{T}\left\|\bar{\varphi}_{i}(t)\right\|_{C_{0}(\omega)} d t .
\end{aligned}
$$

This implies

$$
-\int_{0}^{T}\left\langle\bar{u}_{i}(t), \bar{\varphi}_{i}(t)\right\rangle_{M(\omega), C_{0}(\omega)} d t=\gamma \int_{0}^{T}\left\|\bar{\varphi}_{i}(t)\right\|_{C_{0}(\omega)} d t
$$

and consequently

$$
\int_{0}^{T}\left\langle u(t)+\bar{u}_{i}(t), \bar{\varphi}_{i}(t)\right\rangle_{M(\omega), C_{0}(\omega)} d t=0 .
$$

Moreover, we have for almost every $t \in I$

$$
\left\langle u(t), \bar{\varphi}_{i}(t)\right\rangle_{M(\omega), C_{0}(\omega)}=\gamma\left\|\bar{\varphi}_{i}(t)\right\|_{C_{0}(\omega)} \geq-\left\langle\bar{u}_{i}(t), \bar{\varphi}_{i}(t)\right\rangle_{M(\omega), C_{0}(\omega)} .
$$

Whence we obtain $\left\langle u(t)+\bar{u}_{i}(t), \bar{\varphi}_{i}(t)\right\rangle_{M(\omega), C_{0}(\omega)} \geq 0$. This inequality along with (3.16) yields

$$
-\left\langle\bar{u}_{i}(t), \bar{\varphi}_{i}(t)\right\rangle_{M(\omega), C_{0}(\omega)}=\left\langle u(t), \bar{\varphi}_{i}(t)\right\rangle_{M(\omega), C_{0}(\omega)}=\gamma\left\|\bar{\varphi}_{i}(t)\right\|_{C_{0}(\omega)} .
$$

This identity yields $\left\|\bar{u}_{i}(t)\right\|_{M(\omega)}=\gamma$ if $\bar{\varphi}_{i}(t) \not \equiv 0$ and $-\left\langle\bar{u}_{i}(t), \bar{\varphi}_{i}(t)\right\rangle_{M(\omega), C_{0}(\omega)}=$ $\left\|\bar{u}_{i}(t)\right\|_{M(\omega)}\left\|\bar{\varphi}_{i}(t)\right\|_{C_{0}(\omega)}$ holds. Then, we can apply [9, Lemma 3.4] to get the inclusions (3.14).

Next we define the Lagrangian function associated with the control problem $(\mathrm{P})$. To this end, first we consider the functional $j: M(\omega) \longrightarrow[0, \infty)$ given by $j(u)=$ $\|u\|_{M(\omega)}$. This is a convex and Lipschitz functional having directional derivatives $j^{\prime}(u ; v)$ for all $u, v \in M(\omega)$. To give an expression for the derivative $j^{\prime}(u ; v)$ we consider the Lebesgue decomposition of $v$ with respect to $|u|: v=v_{a}+v_{s}$ with $d v_{a}=g_{v} d|u|$, where $v_{a}$ and $v_{s}$ are the absolutely continuous and singular parts of $v$ with respect to $|u|$, and $g_{v} \in L^{1}(|u|)$ is the Radon-Nikodym derivative of $v$ with respect to $|u|$. We can also write $d u=g_{u} d|u|$ where $g_{u}$ is a measurable function such that $\left|g_{u}(x)\right|=1$ for all $x \in \omega$. Actually, $g_{u}$ is the Radon-Nikodym derivative of $u$ with respect to $|u|$. The reader is referred, for instance, to [25, Chapter 6] for these issues. Now we have the following result taken from [12, Proposition 3.3].

Proposition 3.4. Let $u, v \in M(\omega)$; then

$$
j^{\prime}(u ; v)=\int_{\omega} g_{v} d u+\left\|v_{s}\right\|_{M(\omega)} .
$$

Given $\mathbf{u}, \mathbf{v} \in L^{\infty}(I ; \mathbf{M}(\omega))$, we denote by $g_{v_{i}}(t)$ the Radon-Nikodym derivative of $v_{i}(t)$ with respect to $\left|u_{i}(t)\right|$ and $v_{i s}(t)$ the singular part of $v_{i}(t)$ with respect to $\left|u_{i}(t)\right|$. Then, $g_{v_{i}}: \omega \times I \longrightarrow \mathbb{R}$ is a measurable function.

Associated with the control problem $(\mathrm{P})$ we define the Lagrangian function

$$
\mathcal{L}: L^{\infty}(I ; \mathbf{M}(\omega)) \times L^{1}(I)^{2} \longrightarrow \mathbb{R}, \quad \mathcal{L}(\mathbf{u}, \boldsymbol{\psi})=J(u)+\sum_{i=1}^{2} \int_{0}^{T} \psi_{i}(t) j\left(u_{i}(t)\right) d t .
$$

According to (3.1) and (3.17) the directional derivative of $\mathcal{L}$ with respect to the first variable is given by 


$$
\begin{aligned}
\frac{\partial \mathcal{L}}{\partial \mathbf{u}}(\mathbf{u}, \boldsymbol{\psi}) \mathbf{v}= & \int_{0}^{T}\left\langle\mathbf{v}(t), \boldsymbol{\varphi}_{u}(t)\right\rangle_{\mathbf{M}(\omega), \mathbf{C}_{0}(\omega)} \\
& +\sum_{i=1}^{2} \int_{0}^{T} \psi_{i}(t)\left\{\int_{\omega} g_{v_{i}}(t) d u_{i}(t)+\left\|v_{i s}(t)\right\|_{M(\omega)}\right\} d t \\
= & \sum_{i=1}^{2} \int_{0}^{T}\left\{\int_{\omega} \varphi_{u i}(t) g_{v_{i}}(t) d\left|u_{i}\right|(t)+\psi_{i}(t) \int_{\omega} g_{v_{i}}(t) d u_{i}(t)\right\} d t \\
& +\sum_{i=1}^{2} \int_{0}^{T}\left\{\left\langle v_{i s}(t), \varphi_{u i}(t)\right\rangle_{M(\omega), C_{0}(\omega)}+\psi_{i}(t)\left\|v_{i s}(t)\right\|_{M(\omega)}\right\} d t .
\end{aligned}
$$

Denote by $\overline{\mathbf{u}} \in \mathbf{U}_{\text {ad }}$ a control with associated adjoint state $\bar{\varphi} \in \mathbf{V}^{2,1}(0, T)$ satisfying (3.14). We define the function $\bar{\phi}$ as follows

$$
\bar{\phi}_{i}(t)=\left\{\begin{array}{cl}
1 & \text { if } \bar{\varphi}_{i}(t) \equiv 0, \\
-\frac{\bar{\varphi}_{i}(t)}{\left\|\bar{\varphi}_{i}(t)\right\|_{C_{0}(\omega)}} & \text { if } \bar{\varphi}_{i}(t) \not \equiv 0,
\end{array} \quad \text { for } i=1,2 .\right.
$$

Then, we infer with (3.14) that

$$
\bar{\phi}_{i}(x, t)=\left\{\begin{array}{ll}
+1 & \text { if } x \in \operatorname{Supp}\left(\bar{u}_{i}^{+}(t)\right), \\
-1 & \text { if } x \in \operatorname{Supp}\left(\bar{u}_{i}^{-}(t)\right),
\end{array} \quad \text { for } i=1,2,\right.
$$

and, consequently, $d \bar{u}_{i}(t)=\bar{\phi}_{i}(t) d\left|\bar{u}_{i}(t)\right|$ for $i=1,2$. Using these identities and setting $\mathbf{u}=\overline{\mathbf{u}}$ and $\bar{\psi}_{i}(t)=\left\|\bar{\varphi}_{i}(t)\right\|_{C_{0}(\omega)}, i=1,2$, in (3.18) we obtain the directional derivatives

$$
\begin{aligned}
\frac{\partial \mathcal{L}}{\partial \mathbf{u}}(\overline{\mathbf{u}}, \overline{\boldsymbol{\psi}}) \mathbf{v}= & \sum_{i=1}^{2} \int_{0}^{T}\left\{\int_{\omega} \bar{\varphi}_{i}(t) g_{v_{i}}(t) d\left|\bar{u}_{i}\right|(t)+\left\|\bar{\varphi}_{i}(t)\right\|_{C_{0}(\omega)} \int_{\omega} g_{v_{i}}(t) d \bar{u}_{i}(t)\right\} d t \\
& +\sum_{i=1}^{2} \int_{0}^{T}\left\{\left\langle v_{i s}(t), \bar{\varphi}_{i}(t)\right\rangle_{M(\omega), C_{0}(\omega)}+\left\|\bar{\varphi}_{i}(t)\right\|_{C_{0}(\omega)}\left\|v_{i s}(t)\right\|_{M(\omega)}\right\} d t \\
= & \sum_{i=1}^{2} \int_{0}^{T}\left\{\int_{\omega}\left[\bar{\varphi}_{i}(t)+\left\|\bar{\varphi}_{i}(t)\right\|_{C_{0}(\omega)} \bar{\phi}_{i}(t)\right] g_{v_{i}}(t) d\left|\bar{u}_{i}\right|(t) d t\right. \\
& +\sum_{i=1}^{2} \int_{0}^{T}\left\{\left\langle v_{i s}(t), \bar{\varphi}_{i}(t)\right\rangle_{M(\omega), C_{0}(\omega)}+\left\|\bar{\varphi}_{i}(t)\right\|_{C_{0}(\omega)}\left\|v_{i s}(t)\right\|_{M(\omega)}\right\} d t \\
= & \sum_{i=1}^{2} \int_{0}^{T}\left\{\left\langle v_{i s}(t), \bar{\varphi}_{i}(t)\right\rangle_{M(\omega), C_{0}(\omega)}+\left\|\bar{\varphi}_{i}(t)\right\|_{C_{0}(\omega)}\left\|v_{i s}(t)\right\|_{M(\omega)}\right\} d t .
\end{aligned}
$$

From the above expression we deduce that $\frac{\partial \mathcal{L}}{\partial \mathbf{u}}(\overline{\mathbf{u}}, \overline{\boldsymbol{\psi}})$ can be extended to a linear continuous form $\frac{\partial \mathcal{L}}{\partial \mathbf{u}}(\overline{\mathbf{u}}, \overline{\boldsymbol{\psi}}): L^{q}(I ; \mathbf{M}(\omega)) \longrightarrow \mathbb{R}$. Indeed, taking into account that $\mathbf{V}^{1,2}(0, T) \subset L^{2}\left(I ; \mathbf{C}_{0}(\omega)\right) \subset L^{q^{\prime}}\left(I ; \mathbf{C}_{0}(\omega)\right)$ we have

$$
\left|\frac{\partial \mathcal{L}}{\partial \mathbf{u}}(\overline{\mathbf{u}}, \overline{\boldsymbol{\psi}}) \mathbf{v}\right| \leq\|\overline{\boldsymbol{\varphi}}\|_{L^{q^{\prime}}\left(I ; \mathbf{C}_{0}(\omega)\right)}\|\mathbf{v}\|_{L^{q}(I ; \mathbf{M}(\omega))} \quad \forall \mathbf{v} \in L^{q}(I ; \mathbf{M}(\omega)) .
$$

From the inequality

$$
\left|\left\langle v_{i s}(t), \bar{\varphi}_{i}(t)\right\rangle_{M(\omega), C_{0}(\omega)}\right| \leq\left\|\bar{\varphi}_{i}(t)\right\|_{C_{0}(\omega)}\left\|v_{i s}(t)\right\|_{M(\omega)} \text { for } i=1,2,
$$

Copyright $@$ ㅇ by SIAM. Unauthorized reproduction of this article is prohibited. 
[9, Lemma 3.4], the fact that $v_{i s}(t)$ is singular with respect to $\left|\bar{u}_{i}(t)\right|$, and recalling that $\overline{\boldsymbol{\psi}}(t)=\|\overline{\boldsymbol{\varphi}}(t)\|_{\mathbf{C}_{0}(\omega)}$ we deduce

$$
\begin{aligned}
& \frac{\partial \mathcal{L}}{\partial \mathbf{u}}(\overline{\mathbf{u}}, \overline{\boldsymbol{\psi}}) \mathbf{v} \geq 0 \quad \forall \mathbf{v} \in L^{q}(I ; \mathbf{M}(\omega)), \\
& \frac{\partial \mathcal{L}}{\partial \mathbf{u}}(\overline{\mathbf{u}}, \overline{\boldsymbol{\psi}}) \mathbf{v}=0 \text { if and only if for } i=1 \text { and } 2, \text { in case } \bar{\varphi}_{i}(t) \not \equiv 0 \\
& \left\{\begin{array}{l}
\operatorname{Supp}\left(\bar{v}_{i s}^{+}(t)\right) \subset\left\{x \in \omega \backslash \operatorname{Supp}\left(\left|\bar{u}_{i}(t)\right|\right): \bar{\varphi}_{i}(x, t)=-\left\|\bar{\varphi}_{i}(t)\right\|_{C_{0}(\omega)}\right\}, \\
\operatorname{Supp}\left(\bar{v}_{i s}^{-}(t)\right) \subset\left\{x \in \omega \backslash \operatorname{Supp}\left(\left|\bar{u}_{i}(t)\right|\right): \bar{\varphi}_{i}(x, t)=+\left\|\bar{\varphi}_{i}(t)\right\|_{C_{0}(\omega)}\right\} .
\end{array}\right.
\end{aligned}
$$

4. Second-order optimality conditions. In this section we study the secondorder necessary and sufficient optimality conditions for local optimality. Associated with $\overline{\mathbf{u}}$ of $(\mathrm{P})$ we introduce the cone of critical directions

$$
C_{\overline{\mathbf{u}}}=\left\{\mathbf{v} \in L^{q}(I ; \mathbf{M}(\omega)): v_{i} \text { satisfies }(4.2), i=1,2, \text { and } \frac{\partial \mathcal{L}}{\partial \mathbf{u}}(\overline{\mathbf{u}}, \bar{\psi}) \mathbf{v}=0\right\} .
$$

$$
\text { For a.a. } t \in I: \text { if }\left\|\bar{u}_{i}(t)\right\|_{M(\omega)}=\gamma, \text { then }\left\{\begin{array}{l}
j^{\prime}\left(\bar{u}_{i}(t) ; v_{i}(t)\right) \leq 0 \\
j^{\prime}\left(\bar{u}_{i}(t) ; v_{i}(t)\right)=0 \text { if } \bar{\varphi}_{i}(t) \not \equiv 0 .
\end{array}\right.
$$

Now, we formulate the second-order necessary optimality condition.

TheOrem 4.1. Let $\overline{\mathbf{u}}$ be a local minimum of $(\mathrm{P})$. Then, $J^{\prime \prime}(\overline{\mathbf{u}}) \mathbf{v}^{2} \geq 0$ for all $\mathbf{v} \in C_{\overline{\mathbf{u}}}$ holds.

Proof. Let us take $\mathbf{v} \in C_{\overline{\mathbf{u}}} \cap L^{\infty}(I ; \mathbf{M}(\omega))$. We set

$$
v_{i}(t)=g_{v_{i}}(t) d\left|\bar{u}_{i}(t)\right|+v_{i s}(t) \text { and } d \bar{u}_{i}(t)=g_{\bar{u}_{i}}(t) d\left|\bar{u}_{i}(t)\right|, i=1,2,
$$

where $g_{v_{i}}(t)$ and $g_{\bar{u}_{i}}(t)$ are the corresponding Randon-Nikodym derivatives. We define the sets

$$
\begin{aligned}
& I_{\gamma, i}^{0}=\left\{t \in I:\left\|\bar{u}_{i}(t)\right\|_{M(\omega)}=\gamma \text { and } \bar{\varphi}_{i}(t) \equiv 0\right\}, \\
& I_{\gamma, i}^{+}=\left\{t \in I: \bar{\varphi}_{i}(t) \not \equiv 0\right\}, I_{\gamma, i}=I_{\gamma, i}^{0} \cup I_{\gamma, i}^{+}, \quad i=1,2 .
\end{aligned}
$$

Note further that $I=I_{\gamma, i} \cup\left\{t \in I:\|\bar{u}(t)\|_{M(\omega)}<\gamma\right\}$. From (3.14) it follows that $\left\|\bar{u}_{i}(t)\right\|_{M(\omega)}=\gamma$ for every $t \in I_{\gamma, i}$. Proposition 3.4 and $\mathbf{v} \in C_{\overline{\mathbf{u}}}$ yield

$$
j^{\prime}\left(\bar{u}_{i}(t), v_{i}(t)\right)=\int_{\omega} g_{v_{i}}(t) d \bar{u}_{i}(t)+\left\|v_{i s}(t)\right\|_{M(\omega)}\left\{\begin{array}{l}
=0 \text { if } t \in I_{\gamma, i}^{+}, \quad i=1,2 . \\
\leq 0 \text { if } t \in I_{\gamma, i}^{0},
\end{array}\right.
$$

Let us denote

$$
a(t)=\int_{\omega} g_{v_{i}}(t) d \bar{u}_{i}(t) \text { and } a_{k}(t)=\int_{\omega} \operatorname{proj}_{[-k,+k]}\left(g_{v_{i}}(t)\right) d \bar{u}_{i}(t) \text { for } k \geq 1 .
$$

With (4.3) and Lebesgue's theorem we infer

$$
j^{\prime}\left(\bar{u}_{i}(t) ; v_{i}(t)\right)=a(t)+\left\|v_{i s}(t)\right\|_{M(\omega)}\left\{\begin{array}{l}
=0 \text { if } t \in I_{\gamma, i}^{+}, \\
\leq 0 \text { if } t \in I_{\gamma, i}^{0},
\end{array} \text { and } \lim _{k \rightarrow \infty} a_{k}(t)=a(t)\right.
$$

in the a.e. sense. Now, we set

$$
g_{v_{i}}^{k}(t)=\operatorname{proj}_{[-k,+k]}\left(g_{v_{i}}(t)\right)+\frac{a(t)-a_{k}(t)}{\gamma} g_{\bar{u}_{i}}(t)
$$

Copyright $@$ by SIAM. Unauthorized reproduction of this article is prohibited. 
and

$$
d v_{k, i}(t)=\left\{\begin{array}{cl}
0 & \text { if } \gamma-\frac{1}{k}<\left\|\bar{u}_{i}(t)\right\|_{M(\omega)}<\gamma, \\
g_{v_{i}}^{k}(t) d \bar{u}_{i}(t)+d v_{i s}(t) & \text { if } t \in I_{\gamma, i}, \\
d v_{i}(t), & \text { otherwise. }
\end{array}\right.
$$

Below we shall argue that $\mathbf{v}_{k} \rightarrow \mathbf{v}$ in $L^{q}(I ; \mathbf{M}(\omega))$. From (4.4) we get

$$
\begin{aligned}
j^{\prime}\left(\bar{u}_{i}(t) ; v_{k, i}(t)\right) & =a_{k}(t)+\frac{a(t)-a_{k}(t)}{\gamma} \int_{\omega} g_{\bar{u}_{i}}(t) d \bar{u}_{i}(t)+\left\|v_{i s}(t)\right\|_{M(\omega)} \\
& =a_{k}(t)+\frac{a(t)-a_{k}(t)}{\gamma} \int_{\omega} d\left|\bar{u}_{i}(t)\right|+\left\|v_{i s}(t)\right\|_{M(\omega)} \\
& =a(t)+\left\|v_{i s}(t)\right\|_{M(\omega)}\left\{\begin{array}{l}
=0 \text { if } t \in I_{\gamma, i}^{+}, \\
\leq 0 \text { if } t \in I_{\gamma, i}^{0} .
\end{array}\right.
\end{aligned}
$$

Moreover, from (3.19) we have that $\frac{\partial \mathcal{L}}{\partial \mathbf{u}}(\overline{\mathbf{u}}, \overline{\boldsymbol{\psi}}) \mathbf{v}$ only depends on the singular part of $\mathbf{v}(t)$ with respect to $\overline{\mathbf{u}}(t)$. Since the singular part of $\mathbf{v}_{k}(t)$ is zero or equal to the singular part of $\mathbf{v}(t)$, we conclude that $\frac{\partial \mathcal{L}}{\partial \mathbf{u}}(\overline{\mathbf{u}}, \overline{\boldsymbol{\psi}}) \mathbf{v}_{k}=0$. This identity along with $j^{\prime}\left(\bar{u}_{i}(t) ; v_{k, i}(t)\right)=0$ for $t \in I_{\gamma, i}^{+}, \bar{\varphi}_{i}(t) \equiv 0$ on $I \backslash I_{\gamma, i}^{+}$, and the equality

$$
\frac{\partial \mathcal{L}}{\partial \mathbf{u}}(\overline{\mathbf{u}}, \overline{\boldsymbol{\psi}}) \mathbf{v}_{k}=J^{\prime}(\overline{\mathbf{u}}) \mathbf{v}_{k}+\sum_{i=1}^{2} \int_{0}^{T}\left\|\bar{\varphi}_{i}(t)\right\|_{C_{0}(\omega)} j^{\prime}\left(\bar{u}_{i}(t) ; v_{k, i}(t)\right) d t
$$

imply that $J^{\prime}(\overline{\mathbf{u}}) \mathbf{v}_{k}=0$.

Next we prove that $\overline{\mathbf{u}}+\rho \mathbf{v}_{k} \in \mathbf{U}_{\text {ad }}$ for every $\rho>0$ small enough. Indeed, first we observe that

$$
\left|g_{v, i}^{k}(t)\right| \leq k+\frac{\left|a(t)-a_{k}(t)\right|}{\gamma} \leq k+\frac{2}{\gamma} \int_{\omega}\left|g_{v_{i}}(t)\right| d\left|\bar{u}_{i}(t)\right| \leq k+\frac{2}{\gamma}\|\mathbf{v}\|_{L^{\infty}(I ; \mathbf{M}(\omega))} .
$$

Let us take $\rho_{k}>0$ such that

$$
\rho_{k}\left(1+\frac{2}{\gamma}\right)\|\mathbf{v}\|_{L^{\infty}(I ; \mathbf{M}(\omega))}<\frac{1}{k} .
$$

For $i=1,2$, using (4.4), we deduce for $t \in I_{\gamma, i}$ and $\rho \leq \rho_{k}$

$$
\begin{aligned}
\left\|\bar{u}_{i}(t)+\rho v_{k, i}(t)\right\|_{M(\omega)} & =\int_{\omega}\left|g_{\bar{u}_{i}}(t)+\rho g_{v_{i}}^{k}(t)\right| d\left|\bar{u}_{i}(t)\right|+\rho\left\|v_{i s}(t)\right\|_{M(\omega)} \\
& =\int_{\omega}\left(1+\rho g_{v_{i}}^{k}(t)\right) d \bar{u}_{i}^{+}+\int_{\omega}\left(1-\rho g_{v_{i}}^{k}(t)\right) d \bar{u}_{i}^{-}+\rho\left\|v_{i s}(t)\right\|_{M(\omega)} \\
& =\gamma+\rho\left(\int_{\omega} g_{v_{i}}^{k}(t) d \bar{u}_{i}+\left\|v_{i s}(t)\right\|_{M(\omega)}\right) \\
& =\gamma+\rho\left(a(t)+\left\|v_{i s}(t)\right\|_{M(\omega)}\right) \leq \gamma .
\end{aligned}
$$

Moreover, if $\|\bar{u}(t)\|_{M(\omega)} \leq \gamma-\frac{1}{k}$, then $\left\|\bar{u}(t)+\rho \mathbf{v}_{k}(t)\right\|_{M(\omega)}=\|\bar{u}(t)+\rho \mathbf{v}(t)\|_{M(\omega)} \leq \gamma$ for all $\rho<\rho_{k}$ holds. If $\gamma-\frac{1}{k}<\|\bar{u}(t)\|_{M(\omega)}<\gamma$, then $\left\|\bar{u}(t)+\rho \mathbf{v}_{k}(t)\right\|_{M(\omega)}=$ $\|\bar{u}(t)\|_{M(\omega)}<\gamma$ is fulfilled. Thus, we have that $\overline{\mathbf{u}}+\rho \mathbf{v}_{k} \in \mathbf{U}_{\text {ad }}$ for every $\rho<\rho_{k}$.

Now, using that $\overline{\mathbf{u}}$ is a local minimum of $(\mathrm{P}), J^{\prime}(\overline{\mathbf{u}}) \mathbf{v}_{k}=0$ as proved before, and performing a Taylor expansion we get for $k$ fixed and $\rho$ small enough

$$
\left.0 \leq J\left(\overline{\mathbf{u}}+\rho \mathbf{v}_{k}\right)\right)-J(\overline{\mathbf{u}})=\rho J^{\prime}(\overline{\mathbf{u}}) \mathbf{v}_{k}+\frac{\rho^{2}}{2} J^{\prime \prime}\left(\overline{\mathbf{u}}+\theta \rho \mathbf{v}_{k}\right) \mathbf{v}_{k}^{2}=\frac{\rho^{2}}{2} J^{\prime \prime}\left(\overline{\mathbf{u}}+\theta \rho \mathbf{v}_{k}\right) \mathbf{v}_{k}^{2} .
$$

Copyright $@$ by SIAM. Unauthorized reproduction of this article is prohibited. 
Dividing the expression by $\rho^{2} / 2$, using the fact that $J: L^{q}(I ; \mathbf{M}(\omega)) \rightarrow \mathbb{R}$ is of class $C^{\infty}$, and taking $\rho \rightarrow 0$ we infer $J^{\prime \prime}(\overline{\mathbf{u}}) \mathbf{v}_{k}^{2} \geq 0$. Now, using again Lebesgue's theorem it follows that for almost every $t \in I$

$$
\begin{aligned}
& \lim _{k \rightarrow \infty}\left\|g_{v_{i}}^{k}(t)-g_{v_{i}}(t)\right\|_{L^{1}\left(\left|\bar{u}_{i}(t)\right|\right)}=0 \text { and } \\
& \left\|g_{v_{i}}^{k}(t)-g_{v_{i}}(t)\right\|_{L^{1}\left(\left|\bar{u}_{i}(t)\right|\right)} \leq 2\left\|g_{v_{i}}(t)\right\|_{L^{1}\left(\left|\bar{u}_{i}(t)\right|\right)} \leq 2\left\|v_{i}\right\|_{L^{\infty}(I ; \mathbf{M}(\omega))} .
\end{aligned}
$$

Using these properties we easily obtain that $\mathbf{v}_{k} \rightarrow \mathbf{v}$ in $L^{q}(I ; \mathbf{M}(\omega))$. Then, with Theorem 3.2 we can pass to the limit when $k \rightarrow \infty$ in the above inequality and conclude that $J^{\prime \prime}(\overline{\mathbf{u}}) \mathbf{v}^{2} \geq 0$.

Finally, if $\mathbf{v} \in C_{\overline{\mathbf{u}}} \backslash L^{\infty}(I ; \mathbf{M}(\omega))$, then we take $\left\{\mathbf{v}_{k}\right\}_{k=1}^{\infty} \subset L^{\infty}(I ; \mathbf{M}(\omega))$ defined as follows

$$
\mathbf{v}_{k}(t)=\left\{\begin{array}{cl}
0 & \text { if } \| \mathbf{v}_{k}\left(t \|_{M(\omega)}>k\right. \\
\mathbf{v}(t), & \text { otherwise. }
\end{array}\right.
$$

It is straightforward to check $\mathbf{v}_{k} \in C_{\overline{\mathbf{u}}} \cap L^{\infty}(I ; \mathbf{M}(\omega))$ for every $k \geq 1$ and $\mathbf{v}_{k} \rightarrow \mathbf{v}$ in $L^{q}(I ; \mathbf{M}(\omega))$. Hence, $J^{\prime \prime}(\overline{\mathbf{u}}) \mathbf{v}_{k}^{2} \geq 0$ holds for every $k$, and passing to the limit we obtain $J^{\prime \prime}(\overline{\mathbf{u}}) \mathbf{v}^{2} \geq 0$.

In order to formulate a second-order sufficient condition for local optimality we need to extend the cone of critical directions. Given $(\overline{\mathbf{u}}, \bar{\varphi}) \in \mathbf{U}_{\mathrm{ad}} \times \mathbf{V}^{2,1}(0, T)$ satisfying (3.13)-(3.14), we define for $\tau>0$

$$
\begin{aligned}
C_{\overline{\mathbf{u}}}^{\tau}= & \left\{\mathbf{v} \in L^{q}(I ; \mathbf{M}(\omega)): v_{i} \text { satisfies }(4.6), i=1,2\right. \text {, and } \\
& \left.\frac{\partial \mathcal{L}}{\partial \mathbf{u}}(\overline{\mathbf{u}}, \bar{\psi}) \mathbf{v} \leq \tau\left\|\mathbf{z}_{\mathbf{v}}\right\|_{\mathbf{L}^{2}(Q)}\right\},
\end{aligned}
$$

where $\mathbf{z}_{\mathbf{v}}=G^{\prime}(\overline{\mathbf{u}}) \mathbf{v}$.

$$
\left\{\begin{array}{l}
\text { For a.a. } t \in I: \text { if }\left\|\bar{u}_{i}(t)\right\|_{M(\omega)}=\gamma, \text { then } j^{\prime}\left(\bar{u}_{i}(t) ; v_{i}(t)\right) \leq 0, \\
\text { moreover } \sum_{i=1}^{2} \int_{0}^{T}\left\|\bar{\varphi}_{i}(t)\right\|_{C_{0}(\omega)} j^{\prime}\left(\bar{u}_{i}(t) ; v_{i}(t)\right) d t \geq-\tau\left\|\mathbf{z}_{\mathbf{v}}\right\|_{\mathbf{L}^{2}(Q)} .
\end{array}\right.
$$

The last condition is a relaxation of the second condition of (4.2).

Theorem 4.2. Let $(\overline{\mathbf{u}}, \bar{\varphi}) \in \mathbf{U}_{\mathrm{ad}} \times \mathbf{V}^{2,1}(0, T)$ satisfy (3.13)-(3.14). Assume that

$$
\begin{aligned}
& \exists r \in(4, q] \text { such that } \mathbf{y}_{0} \in \mathbf{B}_{2, \frac{r}{2}}(\Omega)+\mathbf{B}_{p, q}(\Omega) \text { and } \mathbf{y}_{d}, \overline{\mathbf{y}} \in L^{r}\left(I ; \mathbf{L}^{4}(\Omega)\right), \\
& \exists \tau>0 \text { and } \delta>0 \text { such that } J^{\prime \prime}(\overline{\mathbf{u}}) \mathbf{v}^{2} \geq \delta\left\|\mathbf{z}_{\mathbf{v}}\right\|_{\mathbf{L}^{2}(Q)}^{2} \quad \forall \mathbf{v} \in C_{\overline{\mathbf{u}}}^{\tau} .
\end{aligned}
$$

Then, there exist $\kappa>0$ and $\varepsilon>0$ such that

$$
J(\overline{\mathbf{u}})+\frac{\kappa}{2}\left\|\mathbf{y}_{\mathbf{u}}-\overline{\mathbf{y}}\right\|_{\mathbf{L}^{2}(Q)}^{2} \leq J(\mathbf{u}) \quad \forall \mathbf{u} \in \mathbf{U}_{\mathrm{ad}}:\|\mathbf{u}-\overline{\mathbf{u}}\|_{L^{q}\left(I ; \mathbf{W}^{-1, p}(\Omega)\right)} \leq \varepsilon,
$$

where $\overline{\mathbf{y}}=G(\overline{\mathbf{u}})$.

Remark 4.3. Notice that in the proof of Theorem 3.2 the continuous embedding $\mathcal{Y} \subset L^{4}\left(I ; \mathbf{L}^{4}(\Omega)\right)$ was established. Moreover, by Theorem 2.2 we know that for $q \geq 8$ the regularity assumption on $\overline{\mathbf{y}}$ is automatically satisfied with $r=q$ if $\mathbf{y}_{0} \in$ $\mathbf{B}_{2,4}(\Omega)+\mathbf{B}_{p, q}(\Omega)$. If $q \in(4,8)$ the local regularity $\overline{\mathbf{y}}$ in $L^{r}\left(0, t_{0}, \mathbf{L}^{4}(\Omega)\right)$ for some $t_{0} \in(0, T]$ can be established, but we were not able to prove global regularity in this case. The necessity of $r>4$ is explicitly used in Lemmas 4.8 and 4.9. This assumption allows us to establish the $C\left(\bar{I} ; C^{1}(\bar{\Omega})\right)$ regularity of the adjoint states and, consequently, to prove the crucial Lemma 4.11. Notice that Lemma 4.8 below follows from Theorem 2.3 if $q \geq 8$. 
Remark 4.4. Sufficient second-order optimality conditions are essential to prove stability of the optimal states with respect to perturbations in problem data; see, for instance, [13]. They are also used for proving convergence rates of the optimal states in the context of numerical approximations of the control problem; see [10].

In order to prove this theorem we need to establish some lemmas.

Lemma 4.5. There exists a constant $M_{\gamma}$ such that

$$
\left\|\mathbf{y}_{\mathbf{u}}-\overline{\mathbf{y}}\right\|_{\mathcal{Y}} \leq M_{\gamma}\|\mathbf{u}-\overline{\mathbf{u}}\|_{L^{q}\left(I ; \mathbf{W}^{-1, p}(\Omega)\right)} \forall \mathbf{u} \in \mathbf{U}_{\mathrm{ad}} .
$$

Proof. Let $G_{0}: L^{q}\left(I ; \mathbf{W}^{-1, p}(\Omega)\right) \longrightarrow \mathcal{Y}$ be as defined in the proof of Theorem 2.3. Then, from mean value theorem we infer

$$
\begin{aligned}
\left\|\mathbf{y}_{\mathbf{u}}-\overline{\mathbf{y}}\right\|_{\mathcal{Y}} & =\left\|G_{0}\left(\chi_{\omega} \mathbf{u}\right)-G_{0}\left(\chi_{\omega} \overline{\mathbf{u}}\right)\right\|_{\mathcal{Y}} \\
& \leq \sup _{\mathbf{v} \in \mathbf{U}_{\mathrm{ad}}}\left\|G_{0}^{\prime}\left(\chi_{\omega} \mathbf{v}\right)\right\|_{\mathcal{L}\left(L^{q}\left(I ; \mathbf{W}^{-1, p}(\Omega)\right), \mathcal{Y}\right)}\|\mathbf{u}-\overline{\mathbf{u}}\|_{L^{q}\left(I ; \mathbf{W}^{-1, p}(\Omega)\right)} \\
& =M_{\gamma}\|\mathbf{u}-\overline{\mathbf{u}}\|_{L^{q}\left(I ; \mathbf{W}^{-1, p}(\Omega)\right)} .
\end{aligned}
$$

The constant $M_{\gamma}$ is finite; see the proof of [15, Theorem 5.1].

Lemma 4.6. Given $\mathbf{u} \in \mathbf{U}_{\text {ad }}$ and $\mathbf{v} \in L^{q}(I ; \mathbf{M}(\omega))$, we set $\mathbf{z}_{\mathbf{u}, \mathbf{v}}=G^{\prime}(\mathbf{u}) \mathbf{v}$ and $\mathbf{z}_{\mathbf{v}}=G^{\prime}(\overline{\mathbf{u}}) \mathbf{v}$. Then, there exist constants $M_{1}>0$ and $M_{2}>0$ independent of $\mathbf{u}$ and $\mathbf{v}$ such that

$$
\begin{aligned}
& \left\|\mathbf{z}_{\mathbf{u}, \mathbf{v}}-\mathbf{z}_{\mathbf{v}}\right\|_{\mathbf{L}^{2}(Q)} \leq M_{1}\|\mathbf{u}-\overline{\mathbf{u}}\|_{L^{q}\left(I ; \mathbf{W}^{-1, p}(\Omega)\right)}\left\|\mathbf{z}_{\mathbf{v}}\right\|_{\mathbf{L}^{2}(Q)}, \\
& \left\|\mathbf{z}_{\mathbf{u}, \mathbf{v}}\right\|_{\mathbf{L}^{2}(Q)} \leq M_{2}\left\|\mathbf{z}_{\mathbf{v}}\right\|_{\mathbf{L}^{2}(Q)}
\end{aligned}
$$

Proof. According to (2.3), the equations satisfied by $\mathbf{z}_{\mathbf{u}, \mathbf{v}}$ and $\mathbf{z}_{\mathbf{v}}$ are

$$
\begin{aligned}
& \frac{\partial \mathbf{z}_{\mathbf{u}, \mathbf{v}}}{\partial t}-\nu \Delta \mathbf{z}_{\mathbf{u}, \mathbf{v}}+\left(\mathbf{y}_{\mathbf{u}} \cdot \nabla\right) \mathbf{z}_{\mathbf{u}, \mathbf{v}}+\left(\mathbf{z}_{\mathbf{u}, \mathbf{v}} \cdot \nabla\right) \mathbf{y}_{\mathbf{u}}+\nabla \mathfrak{q}_{\mathbf{u}}=\chi_{\omega} \mathbf{v} \\
& \frac{\partial \mathbf{z}_{\mathbf{v}}}{\partial t}-\nu \Delta \mathbf{z}_{\mathbf{v}}+(\overline{\mathbf{y}} \cdot \nabla) \mathbf{z}_{\mathbf{v}}+\left(\mathbf{z}_{\mathbf{v}} \cdot \nabla\right) \overline{\mathbf{y}}+\nabla \overline{\mathfrak{q}}=\chi_{\omega} \mathbf{v}
\end{aligned}
$$

Subtracting both equations and setting $\mathbf{e}=\mathbf{z}_{\mathbf{u}, \mathbf{v}}-\mathbf{z}_{\mathbf{v}}$ and $\mathfrak{q}=\mathfrak{q}_{\mathbf{v}}-\overline{\mathfrak{q}}$ we get

$$
\left\{\begin{array}{l}
\frac{\partial \mathbf{e}}{\partial t}-\nu \Delta \mathbf{e}+\left(\mathbf{y}_{\mathbf{u}} \cdot \nabla\right) \mathbf{e}+(\mathbf{e} \cdot \nabla) \mathbf{y}_{\mathbf{u}}+\nabla \mathfrak{q}=\mathbf{g} \text { in } Q \\
\operatorname{div} \mathbf{e}=0 \text { in } Q, \mathbf{e}=0 \text { on } \Sigma, \mathbf{e}(0)=0 \text { in } \Omega
\end{array}\right.
$$

where $\mathbf{g}=-\left[\left(\mathbf{y}_{\mathbf{u}}-\overline{\mathbf{y}}\right) \cdot \nabla\right] \mathbf{z}_{\mathbf{v}}-\left(\mathbf{z}_{\mathbf{v}} \cdot \nabla\right)\left(\mathbf{y}_{\mathbf{u}}-\overline{\mathbf{y}}\right)$. From [15, Lemma 2.1] we get that $\mathbf{g} \in L^{2}\left(I ; \mathbf{H}^{-1}(\Omega)\right)$. Then, [15, Proposition 2.7] implies that (4.8) has a unique solution $(\mathbf{e}, \mathfrak{q}) \in \mathbf{W}(0, T) \times W^{-1, \infty}\left(I ; L^{2}(\Omega) / \mathbb{R}\right)$. Take $\mathbf{f} \in \mathbf{L}^{2}(Q)$ arbitrary, and let $\varphi \in \mathbf{V}^{2,1}(0, T)$ be the solution of the adjoint state equation (3.3) with $\mathbf{y}_{\mathbf{u}}-\mathbf{y}_{d}$ replaced by $\mathbf{f}$. We have the estimate

$$
\|\boldsymbol{\varphi}\|_{\mathbf{V}^{2,1}(0, T)} \leq C\|\mathbf{f}\|_{\mathbf{L}^{2}(Q)} \quad \forall \mathbf{f} \in \mathbf{L}^{2}(Q) \forall \mathbf{u} \in \mathbf{U}_{\mathrm{ad}}
$$

Then, we have

$$
\begin{aligned}
\int_{0}^{T} \int_{\Omega} \mathbf{f e} d x d t & =\int_{0}^{T} \int_{\Omega}\left[-\frac{\partial \boldsymbol{\varphi}}{\partial t}-\nu \Delta \boldsymbol{\varphi}-\left(\mathbf{y}_{\mathbf{u}} \cdot \nabla\right) \boldsymbol{\varphi}-(\nabla \boldsymbol{\varphi})^{T} \mathbf{y}_{\mathbf{u}}+\nabla \pi\right] \mathbf{e} d x d t \\
& =\int_{0}^{T}\left[\frac{d}{d t}(\mathbf{e}, \boldsymbol{\varphi})_{\mathbf{L}^{2}(\Omega)}+a(\mathbf{e}, \boldsymbol{\varphi})+b\left(\mathbf{y}_{\mathbf{u}}, \mathbf{e}, \boldsymbol{\varphi}\right)+b\left(\mathbf{e}(t), \mathbf{y}_{\mathbf{u}}, \boldsymbol{\varphi}\right)\right] d t \\
& =\int_{0}^{T}\langle\mathbf{g}, \boldsymbol{\varphi}\rangle_{\mathbf{H}^{-1}(\Omega), \mathbf{H}_{0}^{1}(\Omega)} d t=-\int_{0}^{T}\left\{b\left(\mathbf{y}_{\mathbf{u}}-\overline{\mathbf{y}}, \mathbf{z}_{\mathbf{v}}, \boldsymbol{\varphi}\right)+b\left(\mathbf{z}_{\mathbf{v}}, \mathbf{y}_{\mathbf{u}}-\overline{\mathbf{y}}, \boldsymbol{\varphi}\right)\right\} d t
\end{aligned}
$$

Copyright (c) by SIAM. Unauthorized reproduction of this article is prohibited. 
Let us estimate the last integral. To this end we use the embeddings $\mathbf{H}^{2,1}(Q) \subset$ $L^{4}\left(I ; \mathbf{W}^{1,4}(\Omega)\right)$ and $\mathcal{Y} \subset L^{4}\left(I ; \mathbf{L}^{4}(\Omega)\right)$, and estimates (4.10) and (4.13):

$$
\begin{aligned}
\int_{0}^{T}\left|b\left(\mathbf{y}_{\mathbf{u}}-\overline{\mathbf{y}}, \mathbf{z}_{\mathbf{v}}, \boldsymbol{\varphi}\right)\right| d t & =\int_{0}^{T}\left|b\left(\mathbf{y}_{\mathbf{u}}-\overline{\mathbf{y}}, \boldsymbol{\varphi}, \mathbf{z}_{\mathbf{v}}\right)\right| d t \\
& \leq\left\|\mathbf{y}_{\mathbf{u}}-\overline{\mathbf{y}}\right\|_{L^{4}\left(I ; \mathbf{L}^{4}(\Omega)\right)}\|\boldsymbol{\varphi}\|_{L^{4}\left(I ; \mathbf{W}^{1,4}(\Omega)\right)}\left\|\mathbf{z}_{\mathbf{v}}\right\|_{\mathbf{L}^{2}(Q)} \\
& \leq C^{\prime}\|\mathbf{f}\|_{\mathbf{L}^{2}(Q)}\left\|\mathbf{y}_{\mathbf{u}}-\overline{\mathbf{y}}\right\|_{\mathcal{Y}}\left\|\mathbf{z}_{\mathbf{v}}\right\|_{\mathbf{L}^{2}(Q)} \\
& \leq C^{\prime \prime} M_{\gamma}\|\mathbf{f}\|_{\mathbf{L}^{2}(Q)}\|\mathbf{u}-\overline{\mathbf{u}}\|_{L^{q}\left(I ; \mathbf{W}^{-1, p}(\Omega)\right)}\left\|\mathbf{z}_{\mathbf{v}}\right\|_{\mathbf{L}^{2}(Q)} .
\end{aligned}
$$

The term $b\left(\mathbf{z}_{\mathbf{v}}, \mathbf{y}_{\mathbf{u}}-\overline{\mathbf{y}}, \boldsymbol{\varphi}\right)$ is estimated in the same way. Thus, we have

$$
\int_{0}^{T} \int_{\Omega} \mathbf{f e} d x d t \leq M_{1}\|\mathbf{f}\|_{\mathbf{L}^{2}(Q)}\|\mathbf{u}-\overline{\mathbf{u}}\|_{L^{q}\left(I ; \mathbf{W}^{-1, p}(\Omega)\right)}\left\|\mathbf{z}_{\mathbf{v}}\right\|_{\mathbf{L}^{2}(Q)}
$$

for all $\mathbf{f} \in L^{2}(Q)$ and, consequently, (4.11) is fulfilled. Finally, (4.12) follows from (4.11) and the inequality

$$
\left\|\mathbf{z}_{\mathbf{u}, \mathbf{v}}\right\|_{\mathbf{L}^{2}(Q)} \leq\left\|\mathbf{z}_{\mathbf{u}, \mathbf{v}}-\mathbf{z}_{\mathbf{v}}\right\|_{\mathbf{L}^{2}(Q)}+\left\|\mathbf{z}_{\mathbf{v}}\right\|_{\mathbf{L}^{2}(Q)} .
$$

LEMMA 4.7. There exists $\varepsilon_{0}>0$ such that for all $\mathbf{u} \in \mathbf{U}_{\mathrm{ad}}$ with $\|\mathbf{u}-\overline{\mathbf{u}}\|_{L^{q}\left(I ; \mathbf{W}_{0}^{1, p}(\Omega)\right)}$ $\leq \varepsilon_{0}$ the inequality

$$
\left\|\mathbf{y}_{\mathbf{u}}-\overline{\mathbf{y}}\right\|_{\mathbf{L}^{2}(Q)} \leq 2\left\|\mathbf{z}_{\mathbf{u}-\overline{\mathbf{u}}}\right\|_{\mathbf{L}^{2}(Q)}
$$

holds, where $\mathbf{z}_{\mathbf{u}-\overline{\mathbf{u}}}=G^{\prime}(\overline{\mathbf{u}})(\mathbf{u}-\overline{\mathbf{u}})$.

Proof. Let us consider the equations satisfied by $\mathbf{y}_{\mathbf{u}}, \overline{\mathbf{y}}$ and $\mathbf{z}_{\mathbf{u}-\overline{\mathbf{u}}}$ :

$$
\begin{aligned}
& \frac{\partial \mathbf{y}_{\mathbf{u}}}{\partial t}-\nu \Delta \mathbf{y}_{\mathbf{u}}+\left(\mathbf{y}_{\mathbf{u}} \cdot \nabla\right) \mathbf{y}_{\mathbf{u}}+\nabla \mathfrak{p}_{\mathbf{u}}=\chi_{\omega} \mathbf{u}, \\
& \frac{\partial \overline{\mathbf{y}}}{\partial t}-\nu \Delta \overline{\mathbf{y}}+(\overline{\mathbf{y}} \cdot \nabla) \overline{\mathbf{y}}+\nabla \overline{\mathfrak{p}}=\chi_{\omega} \overline{\mathbf{u}}, \\
& \frac{\partial \mathbf{z}_{\mathbf{u}-\overline{\mathbf{u}}}}{\partial t}-\nu \Delta \mathbf{z}_{\mathbf{u}-\overline{\mathbf{u}}}+(\overline{\mathbf{y}} \cdot \nabla) \mathbf{z}_{\mathbf{u}-\overline{\mathbf{u}}}+\left(\mathbf{z}_{\mathbf{u}-\overline{\mathbf{u}}} \cdot \nabla\right) \overline{\mathbf{y}}+\nabla \mathfrak{q}_{\mathbf{u}-\overline{\mathbf{u}}}=\chi_{\omega}(\mathbf{u}-\overline{\mathbf{u}}) .
\end{aligned}
$$

Setting $\mathbf{e}=\mathbf{y}_{\mathbf{u}}-\overline{\mathbf{y}}-\mathbf{z}_{\mathbf{u}-\overline{\mathbf{u}}}$ and $\mathfrak{q}=\mathfrak{p}_{u}-\overline{\mathfrak{p}}-\mathfrak{q}_{\mathbf{u}-\overline{\mathbf{u}}}$, we infer from the above equations

$$
\frac{\partial \mathbf{e}}{\partial t}-\nu \Delta \mathbf{e}+(\overline{\mathbf{y}} \cdot \nabla) \mathbf{e}+(\mathbf{e} \cdot \nabla) \overline{\mathbf{y}}+\nabla \mathfrak{q}=-\left[\left(\mathbf{y}_{\mathbf{u}}-\overline{\mathbf{y}}\right) \cdot \nabla\right]\left(\mathbf{y}_{\mathbf{u}}-\overline{\mathbf{y}}\right) .
$$

Using again [15, Lemma 2.1], we have that $\left[\left(\mathbf{y}_{\mathbf{u}}-\overline{\mathbf{y}}\right) \cdot \nabla\right]\left(\mathbf{y}_{\mathbf{u}}-\overline{\mathbf{y}}\right) \in L^{2}\left(I ; \mathbf{H}^{-1}(\Omega)\right)$ and, hence, $\mathbf{e} \in \mathbf{W}(0, T)$. Arguing as in the proof of Lemma 4.6 and using (4.10) we infer

$$
\begin{aligned}
\|\mathbf{e}\|_{\mathbf{L}^{2}(Q)} & \leq C_{1}\left\|\mathbf{y}_{\mathbf{u}}-\overline{\mathbf{y}}\right\|_{\mathcal{Y}}\left\|\mathbf{y}_{\mathbf{u}}-\overline{\mathbf{y}}\right\|_{\mathbf{L}^{2}(Q)} \\
& \leq C_{2}\|\mathbf{u}-\overline{\mathbf{u}}\|_{L^{q}\left(I ; \mathbf{W}^{-1, p}(\Omega)\right)}\left\|\mathbf{y}_{\mathbf{u}}-\overline{\mathbf{y}}\right\|_{\mathbf{L}^{2}(Q)} .
\end{aligned}
$$

Let us take $0<\varepsilon_{0}<\frac{1}{2 C_{2}}$. Then, we have

$$
\begin{aligned}
\left\|\mathbf{y}_{\mathbf{u}}-\overline{\mathbf{y}}\right\|_{\mathbf{L}^{2}(Q)} & \leq\|\mathbf{e}\|_{\mathbf{L}^{2}(Q)}+\left\|\mathbf{z}_{\mathbf{u}-\overline{\mathbf{u}}}\right\|_{\mathbf{L}^{2}(Q)} \\
& \leq \frac{1}{2}\left\|\mathbf{y}_{\mathbf{u}}-\overline{\mathbf{y}}\right\|_{\mathbf{L}^{2}(Q)}+\left\|\mathbf{z}_{\mathbf{u}-\overline{\mathbf{u}}}\right\|_{\mathbf{L}^{2}(Q)},
\end{aligned}
$$

which implies (4.14). 
Lemma 4.8. Assume that (4.7) holds. Then, there exists $\bar{\varepsilon}>0$ such that $\mathbf{y}_{\mathbf{u}} \in$ $\mathcal{Y} \cap L^{r}\left(I ; \mathbf{L}^{4}(\Omega)\right)$ for every $\mathbf{u} \in B_{\bar{\varepsilon}}(\overline{\mathbf{u}}) \subset L^{q}(I ; \mathbf{M}(\omega))$. Moreover, if $\left\{\mathbf{u}_{k}\right\}_{k=1}^{\infty} \subset B_{\bar{\varepsilon}}(\overline{\mathbf{u}})$ is a sequence converging to $\overline{\mathbf{u}}$ in $L^{q}\left(I ; \mathbf{W}^{-1, p}(\Omega)\right)$, then $\mathbf{y}_{\mathbf{u}_{k}} \rightarrow \overline{\mathbf{y}}$ in $L^{r}\left(I ; \mathbf{L}^{4}(\Omega)\right)$ holds.

Proof. Take $\mathbf{y}_{0}=\mathbf{y}_{N 0}+\mathbf{y}_{S 0} \in \mathbf{B}_{2, \frac{r}{2}}+\mathbf{B}_{p, q}(\Omega)$. The proof is split in three steps. Step I. From [15, Theorem 2.5] we know that the system

$$
\left\{\begin{array}{l}
\frac{\partial \mathbf{y}_{S}}{\partial t}-\nu \Delta \mathbf{y}_{S}+\nabla \mathbf{p}_{S}=\mathbf{f}_{0}+\mathbf{u} \chi_{\omega} \text { in } Q \\
\operatorname{div} \mathbf{y}_{S}=0 \text { in } Q, \mathbf{y}_{S}=0 \text { on } \Sigma, \mathbf{y}_{S}(0)=\mathbf{y}_{S 0} \text { in } \Omega
\end{array}\right.
$$

has a unique solution $\mathbf{y}_{S} \in \mathbf{W}_{q, p}(0, T)$ satisfying

$$
\left\|\mathbf{y}_{S}\right\|_{\mathbf{W}_{q, p}(0, T)} \leq C_{1}\left(\left\|\mathbf{f}_{0}\right\|_{L^{q}\left(I ; \mathbf{W}_{p^{\prime}}(\Omega)^{\prime}\right)}+\|\mathbf{u}\|_{L^{q}\left(I ; \mathbf{W}^{-1, p}(\Omega)\right)}+\left\|\mathbf{y}_{S 0}\right\|_{\mathbf{B}_{p, q}(\Omega)}\right)
$$

for some constant $C_{1}$ independent of $\mathbf{u}$. Since $p \geq \frac{4}{3}$ and $r \leq q$, we have that $\mathbf{y}_{S} \in L^{r}\left(I ; \mathbf{L}^{4}(\Omega)\right)$.

Now, we take $\mathbf{y} \in \mathbf{W}(0, T)$ as the solution of

$$
\left\{\begin{array}{l}
\frac{\partial \mathbf{y}}{\partial t}-\nu \Delta \mathbf{y}+(\mathbf{y} \cdot \nabla) \mathbf{y}+\left(\mathbf{y}_{S} \cdot \nabla\right) \mathbf{y}+(\mathbf{y} \cdot \nabla) \mathbf{y}_{S}+\nabla \mathfrak{p}=-\left(\mathbf{y}_{S} \cdot \nabla\right) \mathbf{y}_{S} \text { in } Q \\
\operatorname{div} \mathbf{y}=0 \text { in } Q, \mathbf{y}=0 \text { on } \Sigma, \mathbf{y}(0)=\mathbf{y}_{N 0} \text { in } \Omega
\end{array}\right.
$$

The existence and uniqueness of $\mathbf{y}$ follows from [15, Proposition 2.7], as well as the estimate

$$
\|\mathbf{y}\|_{\mathbf{W}(0, T)} \leq \hat{\eta}\left(\left\|\mathbf{y}_{S}\right\|_{L^{q}\left(I ; \mathbf{W}_{p}(\Omega)\right)}+\left\|\mathbf{y}_{N 0}\right\|_{\mathbf{B}_{2, \frac{r}{2}}}\right),
$$

where $\hat{\eta}:[0, \infty) \longrightarrow[0, \infty)$ is a nondecreasing function with $\hat{\eta}(0)=0$. Obviously, the solution of (1.1) is given by $\mathbf{y}_{\mathbf{u}}=\mathbf{y}_{S}+\mathbf{y}$. In the sequel, applying the implicit function theorem, we will prove that $\mathbf{y} \in L^{r}\left(I ; \mathbf{L}^{4}(\Omega)\right)$ if $\mathbf{u} \in B_{\bar{\varepsilon}}(\overline{\mathbf{u}})$ for some $\bar{\varepsilon}>0$.

Step II. First, we write $\overline{\mathbf{y}}=\tilde{\mathbf{y}}_{S}+\tilde{\mathbf{y}}$ with $\tilde{\mathbf{y}}_{S}$ and $\tilde{\mathbf{y}}$ solutions of (4.15) and (4.17) with $\mathbf{u}$ and $\mathbf{y}_{S}$ replaced by $\overline{\mathbf{u}}$ and $\tilde{\mathbf{y}}_{S}$, respectively. Let us prove that $\tilde{\mathbf{y}} \in \mathbf{W}_{\frac{r}{2}, 2}(0, T)$. Observe that $\tilde{\mathbf{y}}$ satisfies the Stokes equations

$$
\frac{\partial \tilde{\mathbf{y}}}{\partial t}-\nu \Delta \tilde{\mathbf{y}}+\nabla \tilde{\mathfrak{p}}=\mathbf{g} \text { in } Q
$$

where $\mathbf{g}=-\left(\tilde{\mathbf{y}}_{S} \cdot \nabla\right) \tilde{\mathbf{y}}_{S}-(\tilde{\mathbf{y}} \cdot \nabla) \tilde{\mathbf{y}}-\left(\tilde{\mathbf{y}}_{S} \cdot \nabla\right) \tilde{\mathbf{y}}-(\tilde{\mathbf{y}} \cdot \nabla) \tilde{\mathbf{y}}_{S}$. Then, using the maximal parabolic regularity for the Stokes system, it is enough to prove that $\mathbf{g} \in L^{\frac{r}{2}}\left(I ; \mathbf{H}^{-1}(\Omega)\right)$ to deduce that $\tilde{\mathbf{y}} \in \mathbf{W}_{\frac{r}{2}, 2}(0, T)$. First we observe that (4.7) implies that $\tilde{\mathbf{y}}=\overline{\mathbf{y}}-\tilde{\mathbf{y}}_{S} \in$ $L^{r}\left(I ; \mathbf{L}^{4}(\Omega)\right)$. Let us prove $\left(\tilde{\mathbf{y}}_{S} \cdot \nabla\right) \tilde{\mathbf{y}}_{S} \in L^{\frac{r}{2}}\left(I ; \mathbf{H}^{-1}(\Omega)\right)$. Indeed, given $\mathbf{z} \in \mathbf{H}_{0}^{1}(\Omega)$ we have

$$
\left|\left\langle\left(\tilde{\mathbf{y}}_{S}(t) \cdot \nabla\right) \tilde{\mathbf{y}}_{S}(t), \mathbf{z}\right\rangle\right|=\left|\left\langle\left(\tilde{\mathbf{y}}_{S}(t) \cdot \nabla\right) \mathbf{z}, \tilde{\mathbf{y}}_{S}(t)\right\rangle\right| \leq\left\|\tilde{\mathbf{y}}_{S}(t)\right\|_{\mathbf{L}^{4}(\Omega)}^{2}\|\mathbf{z}\|_{\mathbf{H}_{0}^{1}(\Omega)} .
$$

Then, we have $\left\|\left(\tilde{\mathbf{y}}_{S} \cdot \nabla\right) \tilde{\mathbf{y}}_{S}\right\|_{L^{\frac{r}{2}}\left(I ; \mathbf{H}^{-1}(\Omega)\right)} \leq\left\|\tilde{\mathbf{y}}_{S}\right\|_{L^{r}\left(I ; \mathbf{L}^{4}(\Omega)\right)}^{2}$. In a similar way we get that $\|(\tilde{\mathbf{y}} \cdot \nabla) \tilde{\mathbf{y}}\|_{L^{\frac{r}{2}}\left(I ; \mathbf{H}^{-1}(\Omega)\right)} \leq\|\tilde{\mathbf{y}}\|_{L^{r}\left(I ; \mathbf{L}^{4}(\Omega)\right)}^{2}$ and $\left\|(\tilde{\mathbf{y}} \cdot \nabla) \tilde{\mathbf{y}}_{S}\right\|_{L^{\frac{r}{2}}\left(I ; \mathbf{H}^{-1}(\Omega)\right)}=\|\left(\tilde{\mathbf{y}}_{S}\right.$. $\nabla) \tilde{\mathbf{y}}\left\|_{L^{\frac{r}{2}}\left(I ; \mathbf{H}^{-1}(\Omega)\right)} \leq\right\| \tilde{\mathbf{y}}\left\|_{L^{r}\left(I ; \mathbf{L}^{4}(\Omega)\right)}\right\| \tilde{\mathbf{y}}_{S} \|_{L^{r}\left(I ; \mathbf{L}^{4}(\Omega)\right)}$. All together this leads to

$$
\|\tilde{\mathbf{y}}\|_{\mathbf{W}_{\frac{r}{2}, 2}(0, T)} \leq C_{2}\left(\left(\|\tilde{\mathbf{y}}\|_{L^{r}\left(I ; \mathbf{L}^{4}(\Omega)\right)}+\left\|\tilde{\mathbf{y}}_{S}\right\|_{L^{r}\left(I ; \mathbf{L}^{4}(\Omega)\right)}\right)^{2}+\left\|\mathbf{y}_{N 0}\right\|_{\mathbf{B} 2, \frac{r}{2}(\Omega)}\right) .
$$

Copyright $@$ by SIAM. Unauthorized reproduction of this article is prohibited. 
Step III. We define the mapping

$$
\begin{aligned}
\mathcal{F}: \mathbf{W}_{\frac{r}{2}, 2}(0, T) \times L^{q}\left(I ; \mathbf{W}^{-1, p}(\Omega)\right) \longrightarrow L^{\frac{r}{2}}\left(I ; \mathbf{V}^{\prime}\right) \times \mathbf{B}_{2, \frac{r}{2}}(\Omega) \\
\mathcal{F}(\mathbf{y}, \mathbf{u})=\left(\frac{\partial \mathbf{y}}{\partial t}+A \mathbf{y}+(\mathbf{y} \cdot \nabla) \mathbf{y}\right. \\
\left.\quad+\left(\mathbf{y}_{S}(\mathbf{u}) \cdot \nabla\right) \mathbf{y}+(\mathbf{y} \cdot \nabla) \mathbf{y}_{S}(\mathbf{u})+\left(\mathbf{y}_{S}(\mathbf{u}) \cdot \nabla\right) \mathbf{y}_{S}(\mathbf{u}), \mathbf{y}(0)-\mathbf{y}_{N 0}\right)
\end{aligned}
$$

where $\mathbf{y}_{S}(\mathbf{u})$ is the solution (4.15) and $A: \mathbf{V} \longrightarrow \mathbf{V}^{\prime}$ is given by $\langle A \mathbf{y}, \mathbf{z}\rangle_{\mathbf{V}^{\prime}, \mathbf{V}}=a(\mathbf{y}, \mathbf{z})$. Using [3, Theorem 3] with $\mathbf{X}_{0}=\mathbf{H}^{-1}(\Omega), \mathbf{X}_{1}=\mathbf{H}_{0}^{1}(\Omega), p=\frac{r}{2}, s=\frac{1}{r}$, and $\theta=\frac{3}{4}$ we obtain

$$
\begin{aligned}
& \mathbf{W}_{\frac{r}{2}, 2}(0, T) \subset L^{r}\left(I ;\left(\mathbf{H}^{-1}(\Omega), \mathbf{H}_{0}^{1}(\Omega)\right)_{\frac{3}{4}, 1}\right) \subset L^{r}\left(I ;\left(\mathbf{H}^{-1}(\Omega), \mathbf{H}_{0}^{1}(\Omega)\right)_{\frac{3}{4}, 2}\right) \\
& =L^{r}\left(I ; \mathbf{H}^{\frac{1}{2}}(\Omega)\right) \subset L^{r}\left(I ; \mathbf{L}^{4}(\Omega)\right) .
\end{aligned}
$$

Let us mention that the inequality $\theta<1-s$ is required in [3, Theorem 3]. This inequality is satisfied due to our assumption $r>4$.

Arguing as in Step II, it yields $(\mathbf{y} \cdot \nabla) \mathbf{y}+\left(\mathbf{y}_{S}(\mathbf{u}) \cdot \nabla\right) \mathbf{y}+(\mathbf{y} \cdot \nabla) \mathbf{y}_{S}(\mathbf{u})+\left(\mathbf{y}_{S}(\mathbf{u}) \cdot\right.$ $\nabla) \mathbf{y}_{S}(\mathbf{u}) \in L^{\frac{r}{2}}\left(I ; \mathbf{V}^{\prime}\right)$ for every $\mathbf{y} \in \mathbf{W}_{\frac{r}{2}, 2}(0, T)$. Consequently, $\mathcal{F}$ is well defined. Furthermore, it is a $C^{\infty}$ function. We have that $\mathcal{F}(\tilde{\mathbf{y}}, \overline{\mathbf{u}})=(0,0)$. Moreover, the partial derivative

$$
\begin{aligned}
& \frac{\partial \mathcal{F}}{\partial \mathbf{y}}(\tilde{\mathbf{y}}, \overline{\mathbf{u}}): \mathbf{W}_{\frac{r}{2}, 2}(0, T) \longrightarrow L^{\frac{r}{2}}\left(I ; \mathbf{V}^{\prime}\right) \times \mathbf{B}_{2, \frac{r}{2}}(\Omega), \\
& \frac{\partial \mathcal{F}}{\partial \mathbf{y}}(\tilde{\mathbf{y}}, \overline{\mathbf{u}}) \mathbf{z}=\left(\frac{\partial \mathbf{z}}{\partial t}+A \mathbf{z}+(\overline{\mathbf{y}} \cdot \nabla) \mathbf{z}+(\mathbf{z} \cdot \nabla) \overline{\mathbf{y}}, \mathbf{z}(0)\right),
\end{aligned}
$$

where $\overline{\mathbf{y}}=\tilde{\mathbf{y}}+\tilde{\mathbf{y}}_{S}=\tilde{\mathbf{y}}+\mathbf{y}_{S}(\overline{\mathbf{u}})$, is an isomorphism. Indeed, the injectivity follows from [15, Proposition 2.7]. Let us prove the surjectivity. Given $\left(\mathbf{f}, \mathbf{z}_{0}\right) \in L^{\frac{r}{2}}\left(I ; \mathbf{V}^{\prime}\right) \times$ $\mathbf{B}_{2, \frac{r}{2}}(\Omega)$, we take a sequence $\left\{\left(\mathbf{f}_{k}, \mathbf{z}_{0 k}\right)\right\}_{k=1}^{\infty} \subset L^{\frac{r}{2}}(I ; \mathbf{H}) \times \mathbf{V}$ such that $\left(\mathbf{f}_{k}, \mathbf{z}_{0 k}\right) \rightarrow$ $\left(\mathbf{f}, \mathbf{z}_{0}\right)$ in $L^{\frac{r}{2}}\left(I ; \mathbf{V}^{\prime}\right) \times \mathbf{B}_{2, \frac{r}{2}}(\Omega)$. For every $k$ we consider the equation

$$
\left\{\begin{array}{l}
\frac{\partial \mathbf{z}_{k}}{\partial t}+A \mathbf{z}_{k}+(\overline{\mathbf{y}} \cdot \nabla) \mathbf{z}_{k}+\left(\mathbf{z}_{k} \cdot \nabla\right) \overline{\mathbf{y}}=\mathbf{f}_{k} \text { for a.a. } t \in I, \\
\mathbf{z}_{k}(0)=\mathbf{z}_{0 k} .
\end{array}\right.
$$

Arguing as we did for (3.3), we get that $\mathbf{z}_{k} \in \mathbf{V}^{2,1}(0, T)$. Moreover, using again [15, Proposition 2.7], we have the estimate analogous to (4.18) for $k_{0}$ large enough:

$$
\left\|\mathbf{z}_{k}\right\|_{\mathbf{W}(0, T)} \leq \hat{\eta}\left(\left\|\mathbf{f}_{k}\right\|_{L^{\frac{r}{2}\left(I ; \mathbf{V}^{\prime}\right)}}+\left\|\mathbf{z}_{0 k}\right\|_{\mathbf{B}_{2, \frac{r}{2}}(\Omega)}\right) \leq \hat{\eta}\left(2\left[\|\mathbf{f}\|_{L^{\frac{r}{2}\left(I ; \mathbf{V}^{\prime}\right)}}+\left\|\mathbf{z}_{0}\right\|_{\mathbf{B}_{2, \frac{r}{2}}(\Omega)}\right]\right)
$$

for every $k \geq k_{0}$. Observe that $\mathbf{z}_{k}$ satisfies the Stokes equations

$$
\frac{\partial \mathbf{z}_{k}}{\partial t}+A \mathbf{z}_{k}=\mathbf{g}_{k}
$$

where $\mathbf{g}_{k}=\mathbf{f}_{k}-(\overline{\mathbf{y}} \cdot \nabla) \mathbf{z}_{k}-\left(\mathbf{z}_{k} \cdot \nabla\right) \overline{\mathbf{y}}$. Then, using again the maximal parabolic regularity for the Stokes system we have

$$
\begin{aligned}
\left\|\mathbf{z}_{k}\right\|_{\mathbf{W}_{\frac{r}{2}, 2}(0, T)} & \leq C_{3}\left(\left\|\mathbf{g}_{k}\right\|_{L^{\frac{r}{2}}\left(I ; \mathbf{V}^{\prime}\right)}+\left\|\mathbf{z}_{0 k}\right\|_{\mathbf{B}_{2, \frac{r}{2}}(\Omega)}\right) \\
& \leq C_{3}\left(\left\|\mathbf{f}_{k}\right\|_{L^{\frac{r}{2}\left(I ; \mathbf{V}^{\prime}\right)}}+2\|\overline{\mathbf{y}}\|_{L^{r}\left(I ; \mathbf{L}^{4}(\Omega)\right)}\left\|\mathbf{z}_{k}\right\|_{L^{r}\left(I ; \mathbf{L}^{4}(\Omega)\right)}+\left\|\mathbf{z}_{0 k}\right\|_{\mathbf{B}_{2, \frac{r}{2}}(\Omega)}\right) .
\end{aligned}
$$

Copyright $@$ by SIAM. Unauthorized reproduction of this article is prohibited. 
From [3, Theorem 3], we know that the embedding $\mathbf{W}_{\frac{r}{2}, 2}(0, T) \subset L^{r}\left(I ; \mathbf{L}^{4}(\Omega)\right)$ is compact. Then, we can apply Lions's lemma with $\mathbf{W}_{\frac{r}{2}, 2}(0, T) \subset L^{r}\left(I ; \mathbf{L}^{4}(\Omega)\right) \subset$ $\mathbf{L}^{2}(Q)$ to deduce the existence of a constant $C_{4}$ such that

$$
\left\|\mathbf{z}_{k}\right\|_{L^{r}\left(I ; \mathbf{L}^{4}(\Omega)\right)} \leq \frac{1}{4 C_{3}\|\overline{\mathbf{y}}\|_{L^{r}\left(I ; \mathbf{L}^{4}(\Omega)\right)}}\left\|\mathbf{z}_{k}\right\|_{\mathbf{W}_{\frac{r}{2}, 2}(0, T)}+C_{4}\left\|\mathbf{z}_{k}\right\|_{\mathbf{L}^{2}(Q)} .
$$

The last two inequalities and (4.19) imply that $\left\{\mathbf{z}_{k}\right\}_{k=1}^{\infty}$ is bounded in $\mathbf{W}_{\frac{r}{2}, 2}(0, T)$. Then, taking a subsequence, we have that $\mathbf{z}_{k} \rightarrow \mathbf{z}$ in $\mathbf{W}_{\frac{r}{2}, 2}(0, T)$ with $\frac{\partial \mathcal{F}}{\partial \mathbf{y}}(\tilde{\mathbf{y}}, \overline{\mathbf{u}}) \mathbf{z}=$ $(\mathbf{f}, \mathbf{z}(0))$, which proves the surjectivity. Hence, from the implicit function theorem we conclude the existence of $\bar{\varepsilon}>0$ such that the statement of the lemma is fulfilled.

Lemma 4.9. Assume that (4.7) holds, and let $\bar{\varepsilon}$ be as defined in Lemma 4.8. Then, for every $\mathbf{u} \in B_{\bar{\varepsilon}}(\overline{\mathbf{u}})$ the solution $\boldsymbol{\varphi}_{\mathbf{u}}$ of (3.3) belongs to $C\left(\bar{I} ; \mathbf{C}^{1}(\bar{\Omega})\right)$, and there exists a constant $M_{3}$ continuously depending on $\left\|\mathbf{y}_{\mathbf{u}}\right\|_{L^{r}\left(I ; \mathbf{L}^{4}(\Omega)\right)}$ such that

$$
\left\|\boldsymbol{\varphi}_{\mathbf{u}}\right\|_{C\left(\bar{I} ; \mathbf{C}^{1}(\bar{\Omega})\right)} \leq M_{3}\left\|\mathbf{y}_{\mathbf{u}}-\mathbf{y}_{d}\right\|_{L^{r}\left(I ; \mathbf{L}^{4}(\Omega)\right)} .
$$

Proof. Let us consider the spaces

$$
\begin{aligned}
& \mathbf{X}=\left\{\mathbf{y} \in L^{r}\left(I ; \mathbf{W}^{2,4}(\Omega)\right) \cap W^{1, r}\left(I ; \mathbf{L}^{4}(\Omega)\right): \mathbf{y}=0 \text { on } \Sigma \text { and } \operatorname{div} \mathbf{y}=0 \text { in } Q\right\}, \\
& \Pi=\left\{\pi \in L^{r}\left(I ; W^{1,4}(\Omega)\right): \int_{\Omega} \pi(t) d x=0 \text { for a.a. } t \in I\right\} .
\end{aligned}
$$

Applying [3, Theorem 3] with $\mathbf{X}_{0}=\mathbf{L}^{4}(\Omega), \mathbf{X}_{1}=\mathbf{W}^{2,4}(\Omega), p=r, \frac{1}{r}<s<\frac{1}{4}$, and $\frac{3}{4}<\theta<1-s$, we obtain that

$$
\begin{aligned}
& \mathbf{X} \subset C^{0, s-\frac{1}{r}}\left(\bar{I} ;\left(\mathbf{X}_{0}, \mathbf{X}_{1}\right)_{\theta, 1}\right) \subset C^{0, s-\frac{1}{r}}\left(\bar{I} ;\left(\mathbf{X}_{0}, \mathbf{X}_{1}\right)_{\theta, 4}\right) \\
& =C^{0, s-\frac{1}{r}}\left(\bar{I} ; \mathbf{W}^{2 \theta, 4}(\Omega)\right) \subset C\left(\bar{I} ; \mathbf{C}^{1}(\bar{\Omega})\right)
\end{aligned}
$$

the embedding $\mathbf{X} \subset C\left(\bar{I} ; \mathbf{C}^{1}(\bar{\Omega})\right)$ being compact. We point out that the lower bound $\frac{3}{4}<\theta$ is used to guarantee the continuous embedding $\mathbf{W}^{2 \theta, 4}(\Omega) \subset \mathbf{C}^{1}(\bar{\Omega})$.

Now, for every $t \in[0,1]$ and $\mathbf{u} \in B_{\bar{\varepsilon}}(\overline{\mathbf{u}})$ we define the linear operators:

$$
\begin{aligned}
& L_{t}: \mathbf{X} \times \Pi \longrightarrow L^{r}\left(I ; \mathbf{L}^{4}(\Omega)\right), \\
& L_{t}(\boldsymbol{\varphi}, \pi)=-\frac{\partial \varphi}{\partial t}-\nu \Delta \boldsymbol{\varphi}-t\left[\left(\mathbf{y}_{\mathbf{u}} \cdot \nabla\right) \boldsymbol{\varphi}+(\nabla \boldsymbol{\varphi})^{T} \mathbf{y}_{\mathbf{u}}\right]+\nabla \pi .
\end{aligned}
$$

Using the embedding $\mathbf{X} \subset C\left(\bar{I} ; \mathbf{C}^{1}(\bar{\Omega})\right)$ and the regularity $\mathbf{y}_{\mathbf{u}} \in L^{r}\left(I ; \mathbf{L}^{4}(\Omega)\right)$ established in Lemma 4.8 , it is obvious that $L_{t}$ is linear and continuous. Moreover the inyectivity of $L_{t}$ follows from Theorem 3.2. We prove that $L_{t}$ is an isomorphism for every $t \in[0,1]$. Hence, taking $t=1$, (4.20) will be deduced. Put $E=\left\{t \in[0,1]: L_{t}\right.$ is an isomorphism $\}$. The maximal parabolic regularity property of the Stokes system implies that $0 \in E$. Moreover, $E$ is a relatively open set in $[0,1]$. Indeed, if $t_{0} \in E$ and $t \in[0,1]$ with $\left|t-t_{0}\right|<\varepsilon$ we have

$$
\begin{aligned}
\left\|L_{t}(\boldsymbol{\varphi}, \pi)-L_{t_{0}}(\boldsymbol{\varphi}, \pi)\right\|_{L^{r}\left(I ; \mathbf{L}^{4}(\Omega)\right)} & =\left|t-t_{0}\right|\left\|\left(\mathbf{y}_{\mathbf{u}} \cdot \nabla\right) \boldsymbol{\varphi}+(\nabla \boldsymbol{\varphi})^{T} \mathbf{y}_{\mathbf{u}}\right\|_{L^{r}\left(I ; \mathbf{L}^{4}(\Omega)\right)} \\
& \leq C_{1} \varepsilon\left\|\mathbf{y}_{u}\right\|_{L^{r}\left(I ; \mathbf{L}^{4}(\Omega)\right)}\|\boldsymbol{\varphi}\|_{\mathbf{X}}
\end{aligned}
$$

therefore $\left\|L_{t}-L_{t_{0}}\right\|_{\mathcal{L}\left(\mathbf{X} \times \Pi, L^{r}\left(I, \mathbf{L}^{4}(\Omega)\right)\right.} \leq C \varepsilon\left\|\mathbf{y}_{u}\right\|_{L^{r}\left(I ; \mathbf{L}^{4}(\Omega)\right)}$. Since the set of isomorphisms is an open set, we have that $L_{t}$ is an isomorphism if $\varepsilon$ is small enough. Now, 
we prove that $E$ is closed. Take a sequence $\left\{t_{k}\right\}_{k=1}^{\infty} \subset E$ such that $t_{k} \rightarrow t$. It is enough to prove that $L_{t}$ is surjective to conclude that $t \in E$. Given an arbitrary element $\mathbf{f} \in L^{r}\left(I ; \mathbf{L}^{4}(\Omega)\right)$, we introduce the sequence $\left\{\left(\varphi_{k}, \pi_{k}\right)\right\}_{k=1}^{\infty} \subset \mathbf{X} \times \Pi$ such that $L_{t_{k}}\left(\boldsymbol{\varphi}_{k}, \pi_{k}\right)=\mathbf{f}$. Using the well known estimates for the Stokes system we have

$$
\begin{aligned}
\left\|\left(\boldsymbol{\varphi}_{k}, \pi_{k}\right)\right\| \mathbf{X} \times \Pi & \leq C_{2}\left\|\mathbf{f}+t_{k}\left[\left(\mathbf{y}_{\mathbf{u}} \cdot \nabla\right) \boldsymbol{\varphi}_{k}+\left(\nabla \boldsymbol{\varphi}_{k}\right)^{T} \mathbf{y}_{\mathbf{u}}\right]\right\|_{L^{r}\left(I ; \mathbf{L}^{4}(\Omega)\right)} \\
& \leq C_{2}\left(\|\mathbf{f}\|_{L^{r}\left(I ; \mathbf{L}^{4}(\Omega)\right)}+2\left\|\mathbf{y}_{\mathbf{u}}\right\|_{L^{r}\left(I ; \mathbf{L}^{4}(\Omega)\right)}\left\|\boldsymbol{\varphi}_{k}\right\|_{C\left(\bar{I} ; \mathbf{C}^{1}(\bar{\Omega})\right)}\right) .
\end{aligned}
$$

Using again Lions's lemma with the spaces $X \subset C\left(I ; \mathbf{C}^{1}(\bar{\Omega})\right) \subset \mathbf{L}^{2}(Q)$ we deduce the existence of a constant $C_{3}$ such that

$$
\left\|\left(\boldsymbol{\varphi}_{k}, \pi_{k}\right)\right\|_{\mathbf{X} \times \Pi} \leq C_{2}\left(\left(\|\mathbf{f}\|_{L^{r}\left(I ; \mathbf{L}^{4}(\Omega)\right)}+C_{3}\left\|\mathbf{y}_{\mathbf{u}}\right\|_{L^{r}\left(I ; \mathbf{L}^{4}(\Omega)\right)}\left\|\boldsymbol{\varphi}_{k}\right\|_{\mathbf{L}^{2}(Q)}\right)+\frac{1}{2}\left\|\boldsymbol{\varphi}_{k}\right\| \mathbf{X},\right.
$$

which proves the boundedness of $\left\{\left(\boldsymbol{\varphi}_{h}, \pi_{k}\right)\right\}_{k=1}^{\infty}$ in $\mathbf{X} \times \Pi$. Indeed, the boundedness of $\left\{\boldsymbol{\varphi}_{k}\right\}_{k=1}^{\infty}$ in $\mathbf{L}^{2}(Q)$, actually in $\mathbf{V}^{2,1}(0, T)$, follows from Theorem 3.2. Finally, it is straightforward to pass to the limit in $k$ and to conclude that $\left(\varphi_{k}, \pi_{k}\right) \rightarrow(\varphi, \pi)$ in $\mathbf{X} \times \Pi$ with $L_{t}(\varphi, \pi)=\mathbf{f}$. Hence, $L_{t}$ is also an isomorphism. Since $E$ is nonempty, open, and closed, we conclude that $E=[0,1]$ and, consequently, $\varphi_{\mathbf{u}} \in \mathbf{X}$. The estimate (4.20) follows from the above estimates.

Lemma 4.10. Assume that (4.7) is fulfilled, and let $\bar{\varepsilon}$ be as introduced in Lemma 4.8. Then, for every $\mathbf{u} \in B_{\bar{\varepsilon}}(\overline{\mathbf{u}})$ the inequality

$$
\left\|\boldsymbol{\varphi}_{\mathbf{u}}-\overline{\boldsymbol{\varphi}}\right\|_{C\left(\bar{I} ; \mathbf{C}^{1}(\bar{\Omega})\right)} \leq M_{3}\left(1+2 M_{3}\left\|\mathbf{y}_{\mathbf{u}}-\mathbf{y}_{d}\right\|_{L^{r}\left(I ; \mathbf{L}^{4}(\Omega)\right)}\right)\left\|\mathbf{y}_{\mathbf{u}}-\overline{\mathbf{y}}\right\|_{L^{r}\left(I ; \mathbf{L}^{4}(\Omega)\right)}
$$

holds with $M_{3}$ given by Lemma 4.9 .

Proof. Taking $(\mathbf{e}, \pi)=\left(\boldsymbol{\varphi}_{\mathbf{u}}-\bar{\varphi}, \pi_{\mathbf{u}}-\bar{\pi}\right)$ and subtracting the corresponding equations we get

$-\frac{\partial \mathbf{e}}{\partial t}-\nu \Delta \mathbf{e}-(\overline{\mathbf{y}} \cdot \nabla) \mathbf{e}-(\nabla \mathbf{e})^{T} \overline{\mathbf{y}}+\nabla \pi=\mathbf{y}_{\mathbf{u}}-\overline{\mathbf{y}}+\left[\left(\mathbf{y}_{\mathbf{u}}-\overline{\mathbf{y}}\right) \cdot \nabla\right] \boldsymbol{\varphi}_{\mathbf{u}}+\left(\nabla \boldsymbol{\varphi}_{\mathbf{u}}\right)^{T}\left(\mathbf{y}_{\mathbf{u}}-\overline{\mathbf{y}}\right)$ in $Q$.

Then, applying Lemma 4.9 we get

$$
\begin{aligned}
\left\|\boldsymbol{\varphi}_{\mathbf{u}}-\overline{\boldsymbol{\varphi}}\right\|_{C\left(\bar{I} ; \mathbf{C}^{1}(\bar{\Omega})\right)} & \leq M_{3}\left\|\mathbf{y}_{\mathbf{u}}-\overline{\mathbf{y}}+\left[\left(\mathbf{y}_{\mathbf{u}}-\overline{\mathbf{y}}\right) \cdot \nabla\right] \boldsymbol{\varphi}_{\mathbf{u}}+\left(\nabla \boldsymbol{\varphi}_{\mathbf{u}}\right)^{T}\left(\mathbf{y}_{\mathbf{u}}-\overline{\mathbf{y}}\right)\right\|_{L^{r}\left(I ; \mathbf{L}^{4}(\Omega)\right)} \\
& \leq M_{3}\left(1+2\left\|\boldsymbol{\varphi}_{\mathbf{u}}\right\|_{C\left(\bar{I} ; \mathbf{C}^{1}(\bar{\Omega})\right)}\right)\left\|\mathbf{y}_{\mathbf{u}}-\overline{\mathbf{y}}\right\|_{L^{r}\left(I ; \mathbf{L}^{4}(\Omega)\right)} \\
& \leq M_{3}\left(1+2 M_{3}\left\|\mathbf{y}_{\mathbf{u}}-\mathbf{y}_{d}\right\|_{L^{r}\left(I ; \mathbf{L}^{4}(\Omega)\right)}\right)\left\|\mathbf{y}_{\mathbf{u}}-\overline{\mathbf{y}}\right\|_{L^{r}\left(I ; \mathbf{L}^{4}(\Omega)\right)}
\end{aligned}
$$

Lemma 4.11. If (4.7) holds, then for every $\rho>0$ there exists $\varepsilon_{\rho}>0$ such that

$$
\left|\left[J^{\prime \prime}(\mathbf{u})-J^{\prime \prime}(\overline{\mathbf{u}})\right](\mathbf{u}-\overline{\mathbf{u}})^{2}\right| \leq \rho\left\|\mathbf{z}_{\mathbf{u}-\overline{\mathbf{u}}}\right\|_{\mathbf{L}^{2}(Q)}^{2} \quad \forall \mathbf{u} \in \mathbf{U}_{\mathrm{ad}} \cap \bar{B}_{\varepsilon_{\rho}}(\overline{\mathbf{u}}),
$$

where $\bar{B}_{\varepsilon_{\rho}}(\overline{\mathbf{u}})=\left\{\mathbf{u} \in \mathbf{U}_{\mathrm{ad}}:\|\mathbf{u}-\overline{\mathbf{u}}\|_{L^{q}\left(I ; \mathbf{W}^{-1, p}(\Omega)\right)} \leq \varepsilon_{\rho}\right\}$.

Proof. Let $\bar{\varepsilon}$ be as defined in Lemma 4.8 and take $\mathbf{u} \in \mathbf{U}_{\text {ad }} \cap B_{\bar{\varepsilon}}(\overline{\mathbf{u}})$. Let us set $\mathbf{v}=\mathbf{u}-\overline{\mathbf{u}}, \mathbf{z}_{\mathbf{u}, \mathbf{v}}=G^{\prime}(\mathbf{u}) \mathbf{v}$, and $\mathbf{z}_{\mathbf{v}}=G^{\prime}(\overline{\mathbf{u}}) \mathbf{v}$. According to (3.2) we have

$$
\begin{aligned}
& \left|\left[J^{\prime \prime}(\mathbf{u})-J^{\prime \prime}(\overline{\mathbf{u}})\right] \mathbf{v}^{2}\right| \\
& =\left|\int_{Q}\left[\left|\mathbf{z}_{\mathbf{u}, \mathbf{v}}\right|^{2}-2\left(\mathbf{z}_{\mathbf{u}, \mathbf{v}} \cdot \nabla\right) \mathbf{z}_{\mathbf{u}, \mathbf{v}} \boldsymbol{\varphi}_{\mathbf{u}}\right] d x d t-\int_{Q}\left[\left|\mathbf{z}_{\mathbf{v}}\right|^{2}-2\left(\mathbf{z}_{\mathbf{v}} \cdot \nabla\right) \mathbf{z}_{\mathbf{v}} \overline{\boldsymbol{\varphi}}\right] d x d t\right| \\
& \leq \int_{Q}\left|\mathbf{z}_{\mathbf{u}, \mathbf{v}}+\mathbf{z}_{\mathbf{v}}\right|\left|\mathbf{z}_{\mathbf{u}, \mathbf{v}}-\mathbf{z}_{\mathbf{v}}\right| d x d t+2\left|\int_{Q}\left[\left(\mathbf{z}_{\mathbf{u}, \mathbf{v}}-\mathbf{z}_{\mathbf{v}}\right) \cdot \nabla\right] \boldsymbol{\varphi}_{\mathbf{u}} \mathbf{z}_{\mathbf{u}, \mathbf{v}} d x d t\right| \\
& \quad+2\left|\int_{Q}\left(\mathbf{z}_{\mathbf{v}} \cdot \nabla\right)\left(\boldsymbol{\varphi}_{\mathbf{u}}-\overline{\boldsymbol{\varphi}}\right) \mathbf{z}_{\mathbf{u}, \mathbf{v}} d x d t\right|+2\left|\int_{Q}\left(\mathbf{z}_{\mathbf{v}} \cdot \nabla\right) \overline{\boldsymbol{\varphi}}\left(\mathbf{z}_{\mathbf{u}, \mathbf{v}}-\mathbf{z}_{\mathbf{v}}\right) d x d t\right|
\end{aligned}
$$

Copyright $@$ by SIAM. Unauthorized reproduction of this article is prohibited. 
We estimate the last four integrals. For the first one we use Lemma 4.6 as follows

$$
\begin{aligned}
\int_{Q}\left|\mathbf{z}_{\mathbf{u}, \mathbf{v}}+\mathbf{z}_{\mathbf{v}}\right|\left|\mathbf{z}_{\mathbf{u}, \mathbf{v}}-\mathbf{z}_{\mathbf{v}}\right| d x d t & \leq\left\|\mathbf{z}_{\mathbf{u}, \mathbf{v}}+\mathbf{z}_{\mathbf{v}}\right\|_{\mathbf{L}^{2}(Q)}\left\|\mathbf{z}_{\mathbf{u}, \mathbf{v}}-\mathbf{z}_{\mathbf{v}}\right\|_{\mathbf{L}^{2}(Q)} \\
& \leq 2 M_{2} M_{1}\|\mathbf{u}-\overline{\mathbf{u}}\|_{L^{q}\left(I ; \mathbf{W}^{-1, p}(\Omega)\right)}\left\|\mathbf{z}_{\mathbf{v}}\right\|_{\mathbf{L}^{2}(Q)}^{2}
\end{aligned}
$$

For the second integral we use Lemmas 4.6 and 4.9 to get

$$
\begin{aligned}
& \left|\int_{Q}\left[\left(\mathbf{z}_{\mathbf{u}, \mathbf{v}}-\mathbf{z}_{\mathbf{v}}\right) \cdot \nabla\right] \boldsymbol{\varphi}_{\mathbf{u}} \mathbf{z}_{\mathbf{u}, \mathbf{v}} d x d t\right| \\
& \quad \leq\left\|\boldsymbol{\varphi}_{\mathbf{u}}\right\|_{C\left(I ; \mathbf{C}^{1}(\bar{\Omega})\right)}\left\|\mathbf{z}_{\mathbf{u}, \mathbf{v}}-\mathbf{z}_{\mathbf{v}}\right\|_{\mathbf{L}^{2}(Q)}\left\|\mathbf{z}_{\mathbf{v}}\right\|_{\mathbf{L}^{2}(Q)} \\
& \quad \leq M_{1} M_{3}\|\mathbf{u}-\overline{\mathbf{u}}\|_{L^{q}\left(I ; \mathbf{W}^{-1, p}(\Omega)\right)}\left\|\mathbf{y}_{\mathbf{u}}-\mathbf{y}_{d}\right\|_{L^{r}\left(I ; \mathbf{L}^{4}(\Omega)\right)}\left\|\mathbf{z}_{\mathbf{v}}\right\|_{\mathbf{L}^{2}(Q)}^{2}
\end{aligned}
$$

The third integral is estimated with Lemmas 4.6 and 4.10 as follows

$$
\begin{aligned}
& \left|\int_{Q}\left(\mathbf{z}_{\mathbf{v}} \cdot \nabla\right)\left(\boldsymbol{\varphi}_{\mathbf{u}}-\overline{\boldsymbol{\varphi}}\right) \mathbf{z}_{\mathbf{u}, \mathbf{v}} d x d t\right| \\
& \quad \leq\left\|\boldsymbol{\varphi}_{\mathbf{u}}-\bar{\varphi}\right\|_{C\left(\bar{I} ; \mathbf{C}^{1}(\bar{\Omega})\right)} M_{2}\left\|\mathbf{z}_{\mathbf{v}}\right\|_{\mathbf{L}^{2}(Q)}^{2} \\
& \quad \leq M_{3}\left(1+2 M_{3}\left\|\mathbf{y}_{\mathbf{u}}-\overline{\mathbf{y}}\right\|_{L^{r}\left(I ; \mathbf{L}^{4}(\Omega)\right)}\right)\left\|\mathbf{y}_{\mathbf{u}}-\overline{\mathbf{y}}\right\|_{L^{r}\left(I ; \mathbf{L}^{4}(\Omega)\right)} M_{2}\left\|\mathbf{z}_{\mathbf{v}}\right\|_{\mathbf{L}^{2}(Q)}^{2} .
\end{aligned}
$$

The estimate (4.24) is also valid for the fourth integral just changing $\mathbf{y}_{\mathbf{u}}$ by $\overline{\mathbf{y}}$. Finally, the existence of $\varepsilon_{\rho}$ such that (4.22) holds is an immediate consequence of the above estimates and Lemma 4.8 .

Proof of Theorem 4.2. Using that $G_{0}^{\prime}(\bar{u}) \in \mathcal{L}\left(L^{q}\left(I ; \mathbf{W}^{-1, p}(\Omega)\right), \mathcal{Y}\right)$ we get

$$
\left\|\mathbf{z}_{\mathbf{u}-\overline{\mathbf{u}}}\right\|_{\mathbf{L}^{2}(Q)} \leq C_{\Omega}\left\|\mathbf{z}_{\mathbf{u}-\overline{\mathbf{u}}}\right\|_{\mathcal{Y}} \leq C_{\Omega}\left\|G_{0}^{\prime}(\overline{\mathbf{u}})\right\|\|\mathbf{u}-\overline{\mathbf{u}}\|_{L^{q}\left(I ; \mathbf{W}^{-1, p}(\Omega)\right)} .
$$

From Lemmas 4.8 and 4.9, (4.12), and (4.26) we deduce the existence of a constant $M$ such that for every $\mathbf{u} \in \mathbf{U}_{\mathrm{ad}} \cap \bar{B}_{\overline{\frac{\bar{\varepsilon}}{2}}}(\overline{\mathbf{u}})$ we have

$$
\begin{aligned}
\left|J^{\prime \prime}(\mathbf{u})(\mathbf{u}-\overline{\mathbf{u}})^{2}\right| & \leq\left(1+2\left\|\boldsymbol{\varphi}_{\mathbf{u}}\right\|_{C\left(\bar{I} ; \mathbf{C}^{1}(\bar{\Omega})\right)}\right)\left\|\mathbf{z}_{\mathbf{u}, \mathbf{u}-\overline{\mathbf{u}}}\right\|_{\mathbf{L}^{2}(Q)}^{2} \\
& \leq M\|\mathbf{u}-\overline{\mathbf{u}}\|_{L^{q}\left(I ; \mathbf{W}^{-1, p}(\Omega)\right)}\left\|\mathbf{z}_{\mathbf{u}-\overline{\mathbf{u}}}\right\|_{\mathbf{L}^{2}(Q)} \quad \forall \mathbf{u} \in \mathbf{U}_{\mathrm{ad}} .
\end{aligned}
$$

From Lemma 4.11 we obtain the existence of $\varepsilon_{\delta}>0$ such that

$$
\left|\left[J^{\prime \prime}(\mathbf{u})-J^{\prime \prime}(\overline{\mathbf{u}})\right](\mathbf{u}-\overline{\mathbf{u}})^{2}\right| \leq \frac{\delta}{2}\left\|\mathbf{z}_{\mathbf{u}-\overline{\mathbf{u}}}\right\|_{\mathbf{L}^{2}(Q)}^{2} \quad \forall \mathbf{u} \in \mathbf{U}_{\mathrm{ad}} \cap \bar{B}_{\varepsilon_{\delta}}(\overline{\mathbf{u}}),
$$

where $\delta$ is given in (4.8). We take

$$
\varepsilon=\min \left\{\frac{\bar{\varepsilon}}{2}, \varepsilon_{0}, \varepsilon_{\delta}, \frac{\tau}{2 M}, \frac{1}{C_{\Omega}\left\|G_{0}^{\prime}(\overline{\mathbf{u}})\right\|}\right\} \text { and } \kappa=\min \left\{\frac{\tau}{4}, \frac{\delta}{8}\right\},
$$

where $\varepsilon_{0}$ is given in Lemma 4.7. Now, we prove the inequality (4.9). To this end, we take $\mathbf{u} \in \bar{B}_{\varepsilon}(\overline{\mathbf{u}}) \cap \mathbf{U}_{\mathrm{ad}}$ and distinguish two cases.

Case I: $\mathbf{u}-\overline{\mathbf{u}} \notin C_{\overline{\mathbf{u}}}^{\tau}$. At first we note that if $\left\|\bar{u}_{i}(t)\right\|_{M(\omega)}=\gamma$, taking into account that $\overline{\mathbf{u}}+\rho(\mathbf{u}-\overline{\mathbf{u}}) \in \mathbf{U}_{\text {ad }}$ for every $\rho \in(0,1)$, we have

$$
j^{\prime}\left(\bar{u}_{i}(t) ; u_{i}(t)-\bar{u}_{i}(t)\right)=\lim _{\rho \searrow 0} \frac{j\left(\bar{u}_{i}(t)+\rho\left(u_{i}(t)-\bar{u}_{i}(t)\right)\right)-\gamma}{\rho} \leq 0 \text { for } i=1,2 .
$$

Therefore, if $\mathbf{u}-\overline{\mathbf{u}} \notin C_{\overline{\mathbf{u}}}^{\tau}$, then one (or both) of the two conditions holds:

Copyright $@$ by SIAM. Unauthorized reproduction of this article is prohibited. 


$$
\begin{aligned}
& \text { (I) } \frac{\partial \mathcal{L}}{\partial \mathbf{u}}(\overline{\mathbf{u}}, \bar{\psi})(\mathbf{u}-\overline{\mathbf{u}})>\tau\left\|\mathbf{z}_{\mathbf{u}-\overline{\mathbf{u}}}\right\|_{\mathbf{L}^{2}(Q)}, \\
& \text { (II) }-\tau\left\|\mathbf{z}_{\mathbf{u}-\overline{\mathbf{u}}}\right\|_{\mathbf{L}^{2}(Q)}>\sum_{i=1}^{2} \int_{0}^{T}\left\|\bar{\varphi}_{i}(t)\right\|_{C_{0}(\omega)} j^{\prime}\left(\bar{u}_{i}(t) ; v_{i}(t)\right) d t .
\end{aligned}
$$

If (4.29) holds, then performing a Taylor expansion of $J$ around $\overline{\mathbf{u}}$, using the convexity of $j,(3.14)$ and $\|\mathbf{u}(t)\|_{\mathbf{M}(\omega)} \leq \gamma,(4.26),(4.27)$, and taking into account the definitions of $\varepsilon$ and $\kappa$, we get for some $\theta \in[0,1]$

$$
\begin{aligned}
J(\mathbf{u})-J(\overline{\mathbf{u}}) & \geq \mathcal{L}(\mathbf{u}, \overline{\boldsymbol{\psi}})-\mathcal{L}(\overline{\mathbf{u}}, \overline{\boldsymbol{\psi}}) \geq \frac{\partial \mathcal{L}}{\partial \mathbf{u}}(\overline{\mathbf{u}}, \bar{\psi})(\mathbf{u}-\overline{\mathbf{u}})+\frac{1}{2} J^{\prime \prime}(\overline{\mathbf{u}}+\theta(\mathbf{u}-\overline{\mathbf{u}}))(\mathbf{u}-\overline{\mathbf{u}})^{2} \\
& \geq \tau\left\|\mathbf{z}_{\mathbf{u}-\overline{\mathbf{u}}}\right\|_{\mathbf{L}^{2}(Q)}-\frac{\tau}{2}\left\|\mathbf{z}_{\mathbf{u}-\overline{\mathbf{u}}}\right\|_{\mathbf{L}^{2}(Q)}=\frac{\tau}{2}\left\|\mathbf{z}_{\mathbf{u}-\overline{\mathbf{u}}}\right\|_{\mathbf{L}^{2}(Q)} \geq \frac{\tau}{2}\left\|\mathbf{z}_{\mathbf{u}-\overline{\mathbf{u}}}\right\|_{\mathbf{L}^{2}(Q)}^{2} \\
& \geq \frac{\tau}{8}\left\|\mathbf{y}_{\mathbf{u}}-\overline{\mathbf{y}}\right\|_{\mathbf{L}^{2}(Q)}^{2} \geq \frac{\kappa}{2}\left\|\mathbf{y}_{\mathbf{u}}-\overline{\mathbf{y}}\right\|_{\mathbf{L}^{2}(Q)}^{2} .
\end{aligned}
$$

If (4.30) holds, then we obtain $J^{\prime}(\overline{\mathbf{u}})(\mathbf{u}-\overline{\mathbf{u}})>\tau\left\|\mathbf{z}_{\mathbf{u}-\overline{\mathbf{u}}}\right\|_{\mathbf{L}^{2}(Q)}$ due to (3.20). Then, this inequality, (4.26), and (4.27) yield

$$
\begin{aligned}
J(\mathbf{u})-J(\overline{\mathbf{u}}) & =J^{\prime}(\overline{\mathbf{u}})(\mathbf{u}-\overline{\mathbf{u}})+\frac{1}{2} J^{\prime \prime}(\overline{\mathbf{u}}+\theta(\mathbf{u}-\overline{\mathbf{u}}))(\mathbf{u}-\overline{\mathbf{u}})^{2} \\
& >\tau\left\|\mathbf{z}_{\mathbf{u}-\overline{\mathbf{u}}}\right\|_{\mathbf{L}^{2}(Q)}-\frac{\tau}{2}\left\|\mathbf{z}_{\mathbf{u}-\overline{\mathbf{u}}}\right\|_{\mathbf{L}^{2}(Q)} \geq \frac{\kappa}{2}\left\|\mathbf{y}_{\mathbf{u}}-\overline{\mathbf{y}}\right\|_{\mathbf{L}^{2}(Q)}^{2} .
\end{aligned}
$$

Case II: $\mathbf{u}-\overline{\mathbf{u}} \in C_{\overline{\mathbf{u}}}^{\tau}$. We use $J^{\prime}(\overline{\mathbf{u}})(\mathbf{u}-\overline{\mathbf{u}}) \geq 0$, (4.8), and (4.28) to infer

$$
\begin{aligned}
J(\mathbf{u})-J(\overline{\mathbf{u}}) & =J^{\prime}(\overline{\mathbf{u}})(\mathbf{u}-\overline{\mathbf{u}})+\frac{1}{2} J^{\prime \prime}(\overline{\mathbf{u}}+\theta(\mathbf{u}-\overline{\mathbf{u}}))(\mathbf{u}-\overline{\mathbf{u}})^{2} \\
& \geq \frac{1}{2} J^{\prime \prime}(\overline{\mathbf{u}})(\mathbf{u}-\overline{\mathbf{u}})^{2}+\frac{1}{2}\left[J^{\prime \prime}(\overline{\mathbf{u}}+\theta(\mathbf{u}-\overline{\mathbf{u}}))-J^{\prime \prime}(\overline{\mathbf{u}})\right](\mathbf{u}-\overline{\mathbf{u}})^{2} \\
& \geq \frac{\delta}{2}\left\|\mathbf{z}_{\mathbf{u}-\overline{\mathbf{u}}}\right\|_{\mathbf{L}^{2}(Q)}^{2}-\frac{\delta}{4}\left\|\mathbf{z}_{\mathbf{u}-\overline{\mathbf{u}}}\right\|_{\mathbf{L}^{2}(Q)}^{2}=\frac{\delta}{4}\left\|\mathbf{z}_{\mathbf{u}-\overline{\mathbf{u}}}\right\|_{\mathbf{L}^{2}(Q)}^{2} \\
& \geq \frac{\delta}{16}\left\|\mathbf{y}_{\mathbf{u}}-\overline{\mathbf{y}}\right\|_{\mathbf{L}^{2}(Q)}^{2} \geq \frac{\kappa}{2}\left\|\mathbf{y}_{\mathbf{u}}-\overline{\mathbf{y}}\right\|_{\mathbf{L}^{2}(Q)}^{2},
\end{aligned}
$$

which concludes the proof.

\section{REFERENCES}

[1] F. Abergel and R. Temam, On some control problems in fluid mechanics, Theoret. Comput. Fluid Dynamics, 1 (1990), pp. 303-325.

[2] H. Amann, Linear and Quasilinear Parabolic Problems, vol. 1, Birkhäuser, Boston, 1995.

[3] H. Amann, Linear parabolic problems involving measures, Rev. R. Acad. Cien. Serie A. Mat., 95 (2001), pp. 85-119.

[4] T. Bewley, R. Temam, and M. Ziane, Existence and uniqueness of optimal control to the Navier-Stokes equations, C. R. Acad. Sci. Sér. I Math., 330 (2000), pp. 1007-1011.

[5] F. Boyer AND P. FABrie, Mathematical Tools for the Study of the Incompressible NavierStokes Equations and Related Models, Springer, New York, 2013.

[6] E. CASAS, A review on sparse solutions in optimal control of partial differential equations, SEMA J., 74 (2017), pp. 319-344.

[7] E. Casas and K. Chrysafinos, A review of numerical analysis for the discretization of the velocity tracking problem, in Trends in Differential Equations and Applications, SEMA SIMAI Springer Ser. 8, Springer, Cham, 2016, pp. 51-71.

[8] E. Casas, C. Clason, and K. Kunisch, Approximation of elliptic control problems in measure spaces with sparse solutions, SIAM J. Control Optim., 50 (2012), pp. 1735-1752.

Copyright $@$ by SIAM. Unauthorized reproduction of this article is prohibited. 
[9] E. Casas, C. Clason, and K. Kunisch, Parabolic control problems in measure spaces with sparse solutions, SIAM J. Control Optim., 51 (2013), pp. 28-63.

[10] E. CASAS AND K. ChrYsafinos, Error estimates for the approximation of the velocity tracking problem with bang-bang controls, ESAIM Control Optim. Calc. Var., 23 (2017), pp. 12671291.

[11] E. Casas, R. Herzog, and G. Wachsmuth, Analysis of spatio-temporally sparse optimal control problems of semilinear parabolic equations, ESAIM Control Optim. Calc. Var., 23 (2017), pp. 263-295.

[12] E. Casas And K. Kunisch, Optimal control of semilinear elliptic equations in measure spaces, SIAM J. Control Optim., 52 (2014), pp. 339-364.

[13] E. Casas And K. Kunisch, Optimal control of the $2 d$ stationary Navier-Stokes equations with measure valued controls, SIAM J. Control Optim., 57 (2019), pp. 1328-1354.

[14] E. CASAS AND K. Kunisch, Using sparse control methods to identify sources in linear diffusionconvection equations, Inverse Problems, 35 (2019), 114002.

[15] E. CASAS AND K. Kunisch, Well-posedness of evolutionary Navier-Stokes equations with forces of low regularity on two-dimensional domains, preprint, arXiv:2004.10456 [math.AP], 2020.

[16] J. C. De Los Reyes And R. Griesse, State-constrained optimal control of the threedimensional stationary Navier-Stokes equations, J. Math. Anal. Appl., 343 (2008), pp. 257272.

[17] M. Desai And K. Ito, Optimal controls of Navier-Stokes equations, SIAM J. Control Optim., 32 (1994), pp. 1428-1446.

[18] R. E. Edwards, Functional Analysis, Holt, Rinehart and Winston, New York, 1965.

[19] I. Ekeland And R. Temam, Convex Analysis and Variational Problems, North-HollandElsevier, New York, 1976.

[20] M. D. Gunzburger, Perspectives in flow control and optimization, Advances in Design and Control 5, Society for Industrial and Applied Mathematics (SIAM), Philadelphia, 2003.

[21] R. Herzog, G. Stadler, and G. Wachsmuth, Erratum: Directional sparsity in optimal control of partial differential equations, SIAM J. Control Optim., 53 (2015), pp. 2722-2723.

[22] M. Hinze AND K. Kunisch, Second order methods for optimal control of time-dependent fluid flow, SIAM J. Control Optim., 40 (2001), pp. 925-946.

[23] K. Kunisch, Ph. Trautmann, and B. Vexler, Optimal control of the undamped linear wave equation with measure valued controls, SIAM J. Control Optim., 54 (2016), pp. 1212-1244.

[24] J. L. Lions and E. Magenes, Problèmes aux Limites non Homogènes, vol. 1, Dunod, Paris, 1968.

[25] W. Rudin, Real and Complex Analysis, McGraw-Hill Book Co., London, 1970.

[26] D. Serre, Équations de Navier-Stokes stationnaries avec données peu reguliéres, Ann. Sci. Norm. Sup. Pisa, 10 (1983), pp. 543-559.

[27] H. Sohr, The Navier-Stokes Equations. An Elementary Functional Analytic Approach, Birkhäuser Verlag, Basel, 2001.

[28] R. Temam, Navier-Stokes Equations, North-Holland, Amsterdam, 1979.

[29] H. Triebel, Interpolation Theory, Function Spaces, Differential Operators, North-Holland, Berlin, 1978.

[30] F. Tröltzsch and D. Wachsmuth, Second-order sufficient optimality conditions for the optimal control of Navier-Stokes equations, ESAIM Control Optim. Calc. Var., 12 (2006), pp. 93-119.

Copyright $@$ by SIAM. Unauthorized reproduction of this article is prohibited. 